\title{
Assessment of RANS and DES methods for realistic automotive models
}

DOI:

10.1016/j.compfluid.2016.01.008

\section{Document Version}

Accepted author manuscript

Link to publication record in Manchester Research Explorer

\section{Citation for published version (APA):}

Ashton, N., West, A., Lardeau, S., \& Revell, A. (2016). Assessment of RANS and DES methods for realistic automotive models. Computers and Fluids, 128, 1-15. https://doi.org/10.1016/j.compfluid.2016.01.008

\section{Published in:}

Computers and Fluids

\section{Citing this paper}

Please note that where the full-text provided on Manchester Research Explorer is the Author Accepted Manuscript or Proof version this may differ from the final Published version. If citing, it is advised that you check and use the publisher's definitive version.

\section{General rights}

Copyright and moral rights for the publications made accessible in the Research Explorer are retained by the authors and/or other copyright owners and it is a condition of accessing publications that users recognise and abide by the legal requirements associated with these rights.

\section{Takedown policy}

If you believe that this document breaches copyright please refer to the University of Manchester's Takedown Procedures [http://man.ac.uk/04Y6Bo] or contact uml.scholarlycommunications@manchester.ac.uk providing relevant details, so we can investigate your claim.

\section{OPEN ACCESS}




\title{
Assessment of RANS and DES methods for realistic automotive models
}

\author{
N. Ashton ${ }^{\mathrm{a}}$, A. West ${ }^{\mathrm{b}}$, S. Lardeau ${ }^{\mathrm{b}}$, A. Revell ${ }^{\mathrm{a}}$ \\ ${ }^{a}$ Modelling $\mathcal{E}$ Simulation Centre, School of Mechanical, Aerospace $\mathcal{E}$ Civil Engineering, University of Manchester, U.K \\ ${ }^{b}$ CD-adapco, London, U.K
}

\begin{abstract}
This paper presents a comprehensive investigation of RANS and DES models for the Ahmed car body and a realistic automotive vehicle; the DrivAer model. A variety of RANS models, from the 1-equation Spalart Allmaras model to a low-Reynolds number Reynolds Stress model have shown an inability to consistently correctly capture the flow field for both the Ahmed car body and DrivAer model, with the under-prediction of the turbulence in the initial separated shear layer found as a key deficiency. It has been shown that the use of a hybrid RANS-LES model (in this case, Detached Eddy Simulation) offers an advantage over RANS models in terms of the force coefficients, and general flow field for both the Ahmed car body and the DrivAer model. However, for both cases even at the finest mesh level hybrid RANS-LES methods still exhibited inaccuracies. Suggestions are made on possible improvements, in particular on the use of embedded LES with synthetic turbulence generation. Finally the computational cost of each approach is compared, which shows that whilst hybrid RANS-LES offer a clear benefit over RANS models for automotive relevant flows they do so at a much increased cost.
\end{abstract}

Keywords:

Hybrid RANS-LES, DDES, Ahmed Body, DrivAer, RANS, Separated Flow, Automotive

\section{Introduction}

Computational Fluid Dynamics (CFD) has become one the main design tools for the external aerodynamic design of modern day vehicles. The computational resources necessary for these simulations has become more affordable, and commercial software is now sophisticated enough to handle the often complex geometries that are common in automotive design. For typical automotive configurations a separated wake flow exists behind the car body, which has a major impact on the drag and fuel efficiency of the vehicle. Whilst there are other important areas around and within the car, the ability of a CFD simulation to capture this recirculation region is largely a function of the predictive capability of the turbulence model. For this work, a comparison is made between Reynolds Averaged Navier-Stokes (RANS) models (at both eddy-viscosity and second-moment closure levels) and hybrid RANS-LES methods (for this work, Detached Eddy Simulation (DES)). RANS methods assume that the entire spectrum of turbulence can be

Email address: neil.ashton@manchester.ac.uk (N. Ashton) 
modelled by a set of transport equations which arise from decomposing the turbulence into a mean and fluctuating component around this mean. The result of this decomposition is an additional term in the Navier-Stokes equation, named the Reynolds Stress tensor. This term requires additional modelling to close the set of equations. Whilst this approach has a certain level of empiricism, it is also based on key physical mechanisms observed in canonical flows, and thus aim to model the turbulence at a much lower cost than higher fidelity methods such as Large Eddy Simulation (LES). Hybrid RANS-LES methods attempt to provide a compromise between accuracy and computational expense by only using LES in regions of flow which are challenging to RANS models (such as separated flow) but then use RANS models elsewhere. Compared to a wall-resolved LES this results in a significant saving of computational resources.

Until recently, most validation work for turbulence models in the automotive sector was confined to simple automotive models such as the Ahmed car body [1,2] or MIRA/SAE Reference bodies. These bodies resemble a car in terms of their broad aerodynamic features, but miss some of the features of a complete automotive model. Whilst different turbulence models have been evaluated on full-car models, these are often internal studies that cannot be published for confidentiality reasons, and cannot be verified by other groups because of the control on geometry and experimental data.

This work aims to assess the capability of the current state of the art RANS and hybrid RANS-LES models (in this work, DES) for automotive relevant test cases. The desired outcome is to provide industrial users with the information to make informed choices of whether to use RANS or hybrid RANS-LES approaches, both in terms of their accuracy and computational expense.

In this paper the Ahmed car body, a well established automotive test case that combines geometric simplicity with a comprehensive set of experimental data and prior numerical simulations is firstly studied to provide information on the performance of both RANS and hybrid RANS-LES models. Finally, a realistic car model is evaluated to assess whether the conclusions from the Ahmed car hold true for a complete vehicle and also to assess the predictive capability of these different turbulence modelling approaches for a realistic car model. For this we evaluate the DrivAer automotive model [3, 4], a recent effort by TUM and Audi/BMW to produce an open-source car model based closely on realistic complete car geometries with openly available experimental data. The ultimate aim of this paper is to bring together both academic and industrial type studies to make conclusions that can be relevant to the automotive industry.

This paper is structured as follows. Section 2 outlines the turbulence models and approaches used in this study, including information on the validation procedure for the hybrid RANS-LES models. Section 3 provides information the two test cases studied in this paper, including information on the computational grid, boundary condition and numerical schemes in use. In Section 4 the results for the two test cases are presented, using both RANS and hybrid RANS-LES methods. In this section, there is also an analysis of the computational expense of each approach as well as discussion on possible improvements. Finally Section 5 provides the main conclusions from this work and suggestions for further work. 


\section{Turbulence models}

In this paper, a variety of turbulence modelling approaches are assessed. These models represent some of the most popular RANS models as well as variants of the popular DES approach (as shown in Table 1). One of the central aims of this work is to assess the current state-of-the art RANS models against one of the most well validated and popular hybrid RANS-LES models; DES. Whilst this work cannot claim to be fully comprehensive, it represents the range of models typically found in the majority of CFD codes (both commercial and open source) and therefore provides an assessment of the current state-of-the-art RANS and DES models for automotive related flows.

The authors chose DES to represent the hybrid RANS-LES models as this is the model largely in use by the automotive industry in finite-volume solvers.

\begin{tabular}{|c|c|}
\hline RANS Models & Hybrid RANS-LES models \\
\hline Spalart Allmaras (SA) [5] & SST-DDES [6] \\
\hline Realizable $k-\varepsilon(\mathrm{RKE})[7]$ & SST-IDDES [8] \\
\hline$k-\omega$ SST (SST) [9] & SA-DDES [6] \\
\hline Elliptic Blending $k-\varepsilon v^{2}-f($ B-EVM) [10] & SA-IDDES [11] \\
\hline Elliptic Blending Reynolds Stress Model (EB-RSM) [12] & \\
\hline
\end{tabular}

Table 1: Turbulence models used in this study

The principle behind hybrid RANS-LES methods is that a traditional wall-resolved LES is too expensive for complex high-Reynolds number flows that are influenced by near-wall effects. By applying a RANS model in the boundary layer and using LES outside of this region, the total cell count can by greatly reduced as RANS models do not require the same level of grid resolution in the boundary layer. A comprehensive review of hybrid RANS-LES methods can be found in Frohlich et al. [13], but in this study one particular method is used: DES [6]. This method has become increasingly popular due to its ease of implementation and demonstrated performance for a range of applications.

DES can be seen as a RANS model which performs as a LES sub-grid scale model in regions where the grid is fine enough to support LES content and as a standard RANS models where the grid is not. In this study we investigate both standard DDES [6] as well as the variant, IDDES [11], which aims to improve some of the shortcomings of DDES and add a wall-modelled LES capability. Readers are advised to read the doctoral thesis of Mockett [14] for a comprehensive explanation of DES models and its variants.

\subsection{Validation of DES - Decaying Isotropic Turbulence}

Each DES formulation is validated using Decaying Isotropic Turbulence (DIT) to ensure the correct energy decay and validate the model constants. Whilst this is a simple, idealised test case, it is a useful first case to ensure correct 
levels of dissipation before moving to more complex cases. This is particularly important for commercial CFD codes which tend to have many numerical options (both default and user selectable). The solution domain of the DIT calculation is cubic with length $2 \pi$. The solution domain is meshed with three grids consisting of $32^{3}, 64^{3} \& 128^{3}$ cubic and equidistant cells. Periodic boundary conditions are imposed in each direction. The initial velocity field is set with a suitable instance of isotropic turbulence from the Wray [15] DNS data from the AGARD database. In order to obtain the initial values for other variables such as the pressure and turbulence quantities $(k \& \varepsilon$ etc), a frozen velocity field simulation was conducted, the turbulence variables are solved, which once converged were used as initial conditions for the unsteady decay of turbulence simulation.

Calculations were performed with the commercial finite-volume code STAR-CCM+, developed by CD-Adapco. Additional simulations were conducted using the open-source finite-volume Code_Saturne [16] developed by EDF R\&D.

The temporal discretization is $2^{\text {nd }}$ order, and a fully centred $2^{\text {nd }}$ order scheme is used to spatially discretise the momentum convective terms unless otherwise stated. A $2^{\text {nd }}$ order upwind scheme is applied to the turbulent quantities.

Figures 1(a) \& 1(b) show the value of the model constant $C_{D D E S}$ (which can be seen as an equivalent to the Smagorinsky constant in LES) for each model using the $32^{3}, 64^{3} \& 128^{3}$ grids. The $C_{D D E S}$ values were selected to give the appropriate level of dissipation for each model, although with a constant $C_{D D E S}$ value it is not possible to match the DNS data for every mesh resolution. These values agree with the calibrated values from Ashton et al. [17] as well as those observed by many partners in the EU project ATAAC (Advanced Turbulence Simulation Approaches for the Aerospace Community) [18].

Additionally, a $2^{\text {nd }}$ order upwind scheme and a blended central difference scheme were used to illustrate the importance of using a numerical scheme with low numerical dissipation (Figure 1(c)). It can be seen that using any scheme other than the fully central scheme gives too much numerical dissipation. This can lead to an over-prediction of the separation region for such cases as the 2D wall-mounted hump [17] and the Ahmed car body [19].

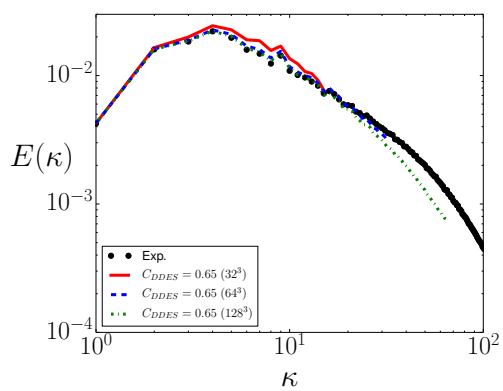

(a)

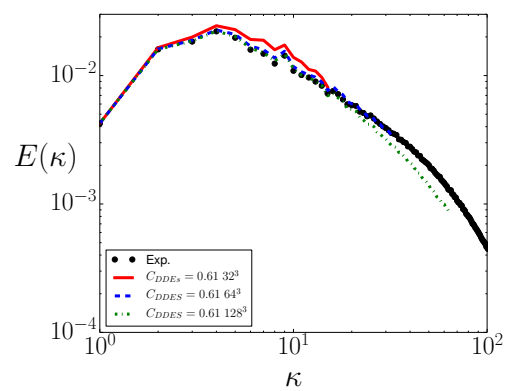

(b)

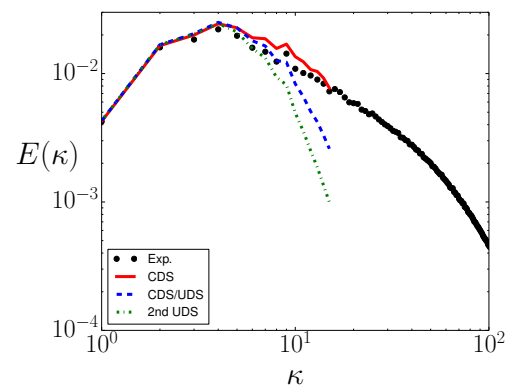

(c)

Figure 1: (a) Calibration of the $C_{D D E S}$ constant using Decaying Isotropic Turbulence for the SA-DDES model (b) Calibration of the $C_{D D E S}$ constant using Decaying Isotropic Turbulence for the SST-DDES model, (c) Numerical scheme sensitivity study using Decaying Isotropic Turbulence for the SA-DDES model 


\section{Test Case Descriptions}

\subsection{Ahmed Car Body}

The Ahmed car body [1][2] represents a generic car geometry with a slanted back and a flat front and has been extensively tested in the literature [20,21, 22, 23, 24]. While it is a much simplified version of a real car, it nevertheless provides many of flow features found in real-life cars such as the complex vortex interactions that occur in its wake and the large 3D separation region behind the car body itself. The wake behind the car body is a result of the interaction between the counter-rotating vortices produced by the slant side edges and the separated flow over the rear of the body (Figure 2(a)). The angle of the slant back section was found to be influential in the structure of the wake and the reattachment point. At $35^{\circ}$ the counter-rotating vortices are weaker, which results in the flow being completely separated over the entire slant back of the vehicle. At $25^{\circ}$ the counter-rotating vortices are strong enough to help to bring enough momentum into the separation region to reattach the flow half way down the slant back.

This case has been the focus of several CFD investigations [20, 21, 22, 25, 26, 27, 19] where a range of RANS, LES and hybrid RANS-LES models were investigated. The general conclusions from the studies involving RANS models $[20,21,25,27,19]$ were dependant on the slant angle. At $35^{\circ}$ (where the separation occurred along the entire slant back) most of the RANS approaches (both simple linear $k-\varepsilon$ and more complex Reynolds stress models) captured the correct level of the turbulent stresses and, as a result, showed good agreement with the experimental results for the separation and reattachment points. However at $25^{\circ}$ (where the complex interaction between the counter-rotating vortices and the separated flow results in a shorter separation region) the majority of RANS models,regardless of mesh refinement or wall treatment, failed to predict the flow correctly. In general, they either failed to predict separation completely, or even when they did, they did not predict the correct separation point and thus were unable to capture the correct size of the recirculation region due to an under-prediction of the turbulent stresses [22].

More recently, a number of LES \& DES studies have been performed on this case, mainly at the more challenging $25^{\circ}$ slant angle $[22,28,29,30,26,31,32,27,19]$. These studies were performed with a range of sub-grid scale models and wall-treatments and, while some were more successful than others, many failed to capture fully the correct recirculation region. The high-Reynolds number $\left(R e=7.68 \times 10^{5}\right)$ meant that even with meshes of up to 48 million cells, the resolution requirements were still not ideal for a wall-resolved LES.

\subsubsection{Computational grid and boundary conditions}

The car body was mounted on four stilts $z / H=0.174$ (where $H=0.288 m$ is the height of the body) above the ground in the experiment to model the effect of the car's height with wheels (and thus capture the important ground effect). Both the $25^{\circ}$ and $35^{\circ}$ slant back angles have been investigated in this study to assess the capability of each model to capture the important changes in flow physics and separation size \& strength. The body has a length of $L / H=3.625$, a height of $H$ and a width of $W / H=1.35$. The flow is at a Reynolds number of $\operatorname{Re}=768,000$ based on the body height $H$ and the free-stream velocity $U_{\infty}=40 \mathrm{~ms}^{-1}$. For both cases, an inlet condition is imposed 


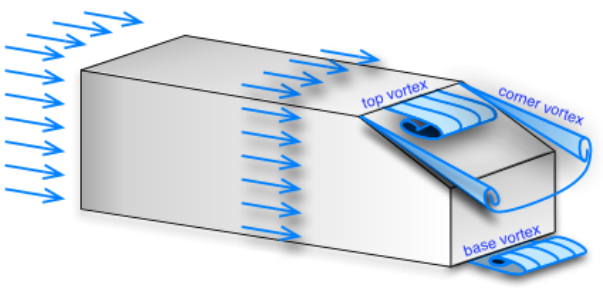

(a)

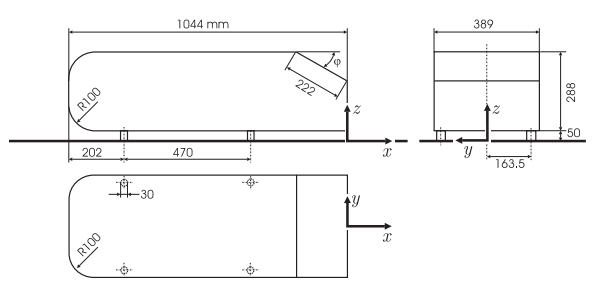

(b)

Figure 2: (a) Ahmed car body flow physics and (b) Ahmed car body dimensions

at $x / H=-7.3$ upstream of the body and an outlet condition is imposed $x / H=20.3$ downstream. A no-slip wall condition is imposed on the ground floor and car body, with slip conditions applied to the wind tunnel walls $(z / H=4.8$ above and $y / H=3.1$ to the side walls). Inlet turbulence levels were found to be largely insensitive due to the large distance between the inlet and car body, thus the turbulent intensity was set to $1 \%$, and $\omega$ and $\varepsilon$ were computed by assuming the turbulent viscosity ratio $v_{t}=10$.

For the $25^{\circ}$ angle, a structured 16 million cell mesh was used (Figure 3(a)); courtesy of Professor Krajnovic of Chalmers University of Technology. For this mesh, the first near-wall cells over the car body had a $y^{+}<1$ and the refinement was concentrated on the near-wall and separation regions.

For the $35^{\circ}$ angle, an unstructured polyhedral based mesh (Figure 3(b)), created in STAR-CCM+ was used with three levels of refinement (Coarse, Medium \& Fine). A constant refinement was kept in the near-wall region for each of these variants, ensuring a $y^{+}<1$ for all near-wall cells. The refinement between the successive meshes was concentrated in the rear separation region over the rear window and behind the car. Mesh size were approximately 4, 10 and 19 million cells for the coarse, medium and fine meshes respectively and it was found that acceptable mesh convergence was reached by the fine mesh.

Calculations were performed using the open-source software Code_Saturne v3.0.1 [33] [16] developed by EDF R\&D and STAR-CCM+ v9.04, developed by CD-adapco. The computational details for each code is shown in Table 2.

\begin{tabular}{|c|c|c|c|c|}
\hline Code & Method & Spatial Scheme & Temporal Scheme & Criteria \\
\hline STAR-CCM+ & RANS & $2^{\text {nd }}$ order Upwind & Steady & $<1 \times 10^{-5}$ residuals \\
STAR-CCM+ & DDES & Hybrid CDS/2 ${ }^{\text {nd }}$ UDS [34] & $2^{\text {nd }}$ order $\left(\Delta t U / L=1 \times 10^{-4}\right)$ & 30 flow units \\
Code Saturne & URANS & $75 \%$ CDS-25\% UDS & $2^{\text {nd }}$ order $\left(\Delta t U / L=1 \times 10^{-4}\right)$ & 10 flow units \\
Code Saturne & DDES & Hybrid CDS $/ 2^{\text {nd }}$ UDS [17] & $2^{\text {nd }}$ order $\left(\Delta t U / L=1 \times 10^{-4}\right)$ & 30 flow units \\
\hline
\end{tabular}




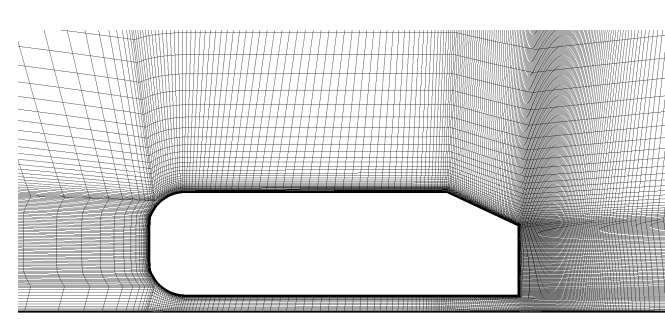

(a)

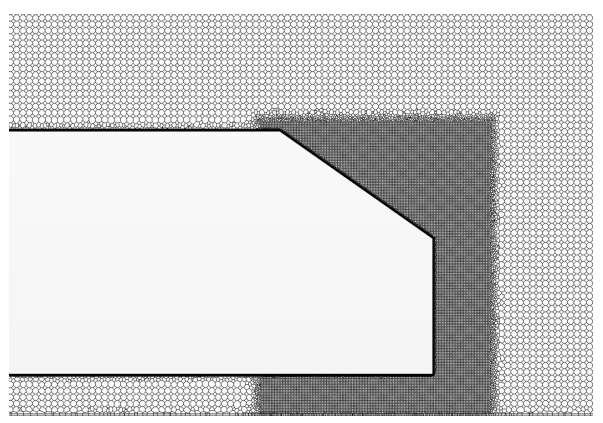

(b)

Figure 3: Meshes for the Ahmed car body (a) Structured 16 million cell mesh for 25 degrees case and (b) unstructured 10 million cell mesh for 35 degrees case.

\subsection{DrivAer automotive model}

A recent addition to the openly available car geometries is the DrivAer model $[3,4]$. In these experiments three car configurations (shown in Figure 4(a)) were subject to a wind tunnel investigation, where global loads were obtained as well as surface pressure information in key areas over and under the vehicles. These three configurations represent three typical car configurations i.e. estate, fastback and notchback. For each configuration, several variants were also investigated where several components were tested on and off the model (side mirrors and wheels) as well as a smooth and detailed underbody. In addition each configuration were tested with and without a rolling road to test the effect of ground simulation. Previous numerical simulations by TUM focused only on the fastback configuration with a single turbulence model [3]. Whilst some effort was made to ensure mesh convergence and to investigate the effect of the several geometry variations, no investigation was made into different turbulence models or the other two DrivAer configurations. The turbulence model in question, the $k-\omega$ SST [9] model was used in the study of [3] and also in this research. The model in [3] used a mesh that did not resolve the near-wall region which makes any direct comparison difficult, however some key flow areas, such as the bonnet/windscreen intersection, show the same trend between the previously mentioned work and the authors previous work. Whilst they showed excellent agreement for the drag coefficient, the pressure distribution over the top and bottom of the car differs from the experiment suggesting that a certain amount of error cancellation is present. This was also observed in recent work using OVERFLOW, which used overset structured meshes [35]. Even though a high quality structured mesh was used, the same discrepancies with the pressure coefficient were observed, which suggests a consistent turbulence modelling error. Guilmineau [36] recently applied DES models to the DrivAer model, one of the first examples of DES being applied to this case. Unfortunately only the fastback configuration was studied, however the findings were in agreement with the previous studies. RANS models (including an Explicit Algebraic RSM model) were unable to correctly predict the forces, particularly the lift force which was considerably different than the experimental data. They found that DES provided much improved accuracy (particularly for the lift force) although there were still inaccuracies compared to the experimental data. 


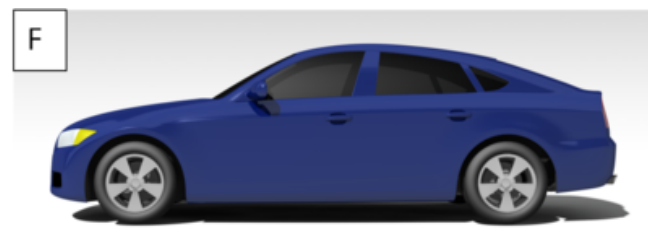

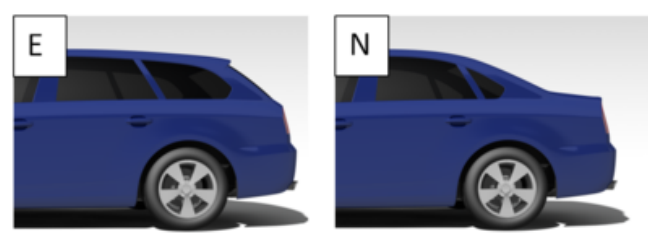

(a)

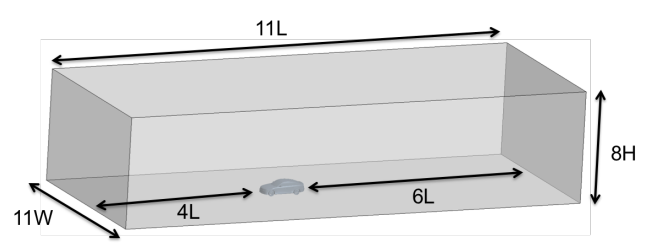

(b)

Figure 4: (a) DrivAer models, F-Fastback, E-Estate, N-Notchback (b) DrivAer computational domain Size

\subsection{Computational Set-up}

\subsubsection{Computational Grid and Boundary Conditions}

Two configurations of the DrivAer model are investigated: the Estate and Fastback variants (Figure 4(a)) both with side mirrors and a smooth underbody. For each configuration the meshing methodology is identical in an attempt to ascertain the predictive performance of different turbulence models with the mesh being a known source of error. A schematic of the domain and car is shown in Figure 4(b), the top of the domain (where a slip wall condition is imposed) is at a height of $8 H$, where $H$ is the height of the car body. An inlet condition is imposed at the start of the domain ( $4 L$ upstream of the car) with a freestream velocity of $40 \mathrm{~ms}^{-1}\left(\operatorname{Re}=1.48 \times 10^{6}\right.$ based upon the car height $H=0.567 m$ ), turbulent viscosity ratio of 20 and a turbulent intensity of $1 \%$. The appropriate values for $k, \varepsilon, \omega$ and were then derived from these values using the appropriate relationship (e.g. $v_{t}=k / \omega$ ). An outlet condition is imposed $6 L$ downstream of the rear of the car. The width of the domain is $11 W$ ( $W$ is the width of the car), where slip wall conditions are imposed. The floor was set to a no-slip condition with non-rotating wheels to match the experimental non-ground simulation case. This was chosen as it removes any rotating tyre modeling error and was also shown to have little effect on the flow physics [4]. The choice of numerics and time-step are described below and summarized in Table 3.

\subsubsection{STAR-CCM+ : RANS}

A steady coupled incompressible finite-volume solver is used for each RANS calculation. A second order upwind scheme is used to discretize the convection and diffusion terms of the momentum and turbulence equations. An Algebraic Multigrid (AMG) method using a V cycle for the momentum equations and a flex cycle for the turbulence quantities was employed. The solution was initialized using a Grid Sequencing Initialization (GSI) method, which solves a fully implicit incomplete-Newton solution algorithm to compute a first-order inviscid flow solution on a series of coarse grids, which provides a better initial flow field than specifying constant values for the flow variables over 
the domain. All simulations were run until the standard deviation of the drag and lift coefficients dropped below $2 \times 10^{-5}$ over 400 samples. This criteria was in conjunction with the residual error for the momentum and turbulent equations reaching a monotonic state below $1 \times 10^{-5}$. Both of these were used to ensure that the flow had a reached full convergence, which typically occurred after 2000-5000 iterations depending on the turbulence model.

\subsubsection{STAR-CCM+ : DDES}

An unsteady segregated incompressible finite-volume solver was used for all the DES computations. A hybrid numerical scheme [34] was used to discretize the convective terms of the momentum equations, which switches between a bounded Central Differencing Scheme (CDS) in regions where LES is active to a 2nd order upwind scheme where RANS modes are active. For the turbulent quantities, a second order upwind scheme is used. The temporal discretization was achieved using a 2nd order Crank-Nicholson type scheme with a non-dimensional time-step of $\Delta t U / L=1 \times 10^{-3}$, ensuring a CFL $<1$ throughout the LES regions. An Algebraic Multigrid (AMG) method using a $\mathrm{V}$ cycle for the momentum equations and a flex cycle for the turbulence quantities was employed. The solution was initialized using the steady RANS results which shortened the time to reach a suitable time for time-averaging. Each simulation was run for 10 convective flow units $(10 \times L / U)$ followed by a further 20 convective units for the gathering of time-averaged statistics.

\begin{tabular}{|c|c|c|c|c|}
\hline Code & Method & Spatial Scheme & Temporal Scheme & Criteria \\
\hline STAR-CCM+ & RANS & $2^{\text {nd }}$ order Upwind & Steady & $<1 \times 10^{-5}$ residuals \\
STAR-CCM+ & DDES & Hybrid CDS $/ 2^{\text {nd }}$ UDS [34] & $2^{\text {nd }}$ order $\left(\Delta t U / L=1 \times 10^{-3}\right)$ & 30 flow units \\
\hline
\end{tabular}

Table 3: Numerical setup for each code and turbulence modelling approach for the DrivAer model.

Five grids were generated in total, for which two were used for the DES calculations. These are summarized in Table 4 and illustrated in Figure 5. The meshes are split into broadly polyhedral/prismatic based for the RANS studies and hexahedral/prismatic cells for the DES studies. Each of the meshes were successively refined to establish mesh convergence, each with greater refinement throughout the rear of the domain (except for a constant highly resolved near-wall mesh, with 20 prism layer cells to ensure the boundary layer was well captured).

\section{Results}

To achieve the objective of both comparing a simple and realistic car model and also assessing the potential benefits of hybrid RANS-LES methods over RANS models, we discuss the performance of the RANS models for both cases before moving on the performance of the hybrid RANS-LES models. It will be seen that there is a common theme between the simple and realistic car cases for both RANS and hybrid RANS-LES models. 


\begin{tabular}{|c|ccc|}
\hline Mesh Name & Mesh Type & $y^{+}$ & Cell Count \\
\hline RANS coarse & Polyhedral+prism & $<1$ & $18 \times 10^{6}$ \\
RANS Medium & Polyhedral+prism & $<1$ & $37 \times 10^{6}$ \\
RANS fine & Polyhedral+prism & $<1$ & $80 \times 10^{6}$ \\
\hline DES Coarse & Polyhedral+prism & $<1$ & $80 \times 10^{6}$ \\
DES Fine & Hexahedral+prism & $<1$ & $100 \times 10^{6}$ \\
\hline
\end{tabular}

Table 4: Mesh type and resolution used within this study.

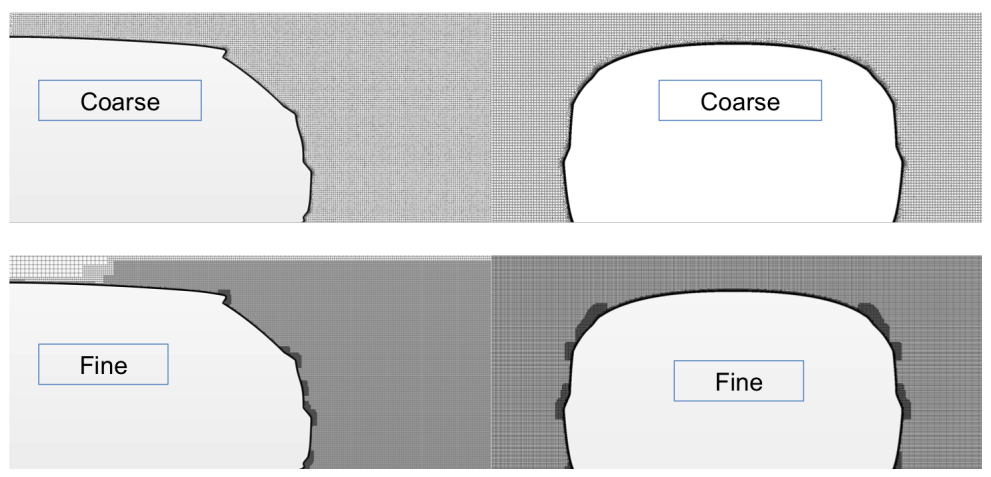

Figure 5: Slices through the symmetry plane of the meshes used for the DES calculations (estate configuration)

\subsection{RANS}

\subsubsection{Ahmed car body: RANS}

Figure 6 show the results for a selection of RANS models for the Ahmed car body with a $35^{\circ}$ rear window angle for both the streamwise velocity and turbulent kinetic energy (TKE). These results were obtained using STAR-CCM+, where a mesh convergence study resulted in a final mesh of 10 million cells. Whilst further mesh refinement may result in some modification of the results, the authors believe that the trends will most likely be very similar. It can be seen from these figures that the models predict largely the correct flow along the symmetry plane which also reflected in other planes. Each model shows good agreement with the experimental data for the TKE, it is also notable that there are only small differences between all the turbulence models, even for a fully separated flow. This is in agreement with many previous studies, including the ERCOFTAC workshops [20,21] using a variety of different turbulence models and grid resolution.

Figure 7 shows the streamwise velocity and turbulent kinetic energy for the Ahmed car body with a $25^{\circ}$ rear window angle. None of the RANS models tested correctly predicted the streamwise velocity with each model overpredicting the size of the recirculation region. It can be seen that this a result of the turbulent kinetic energy being under predicted in the initial separated shear layer (just after separation) which means less turbulent mixing and thus a greater recirculation region. It is again observed that there is little difference between the models tested. These re- 


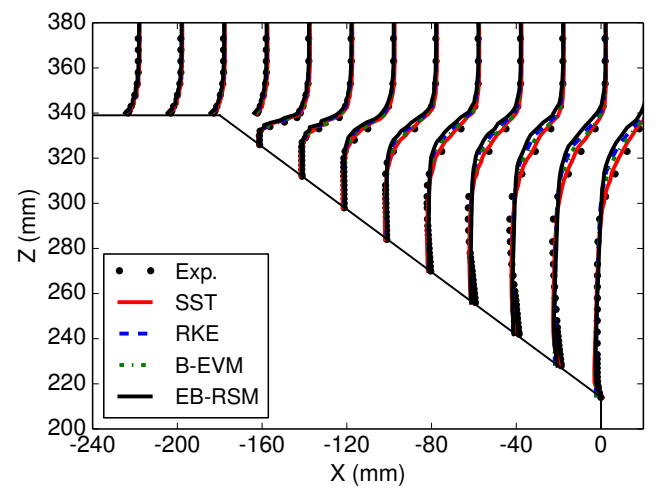

(a)

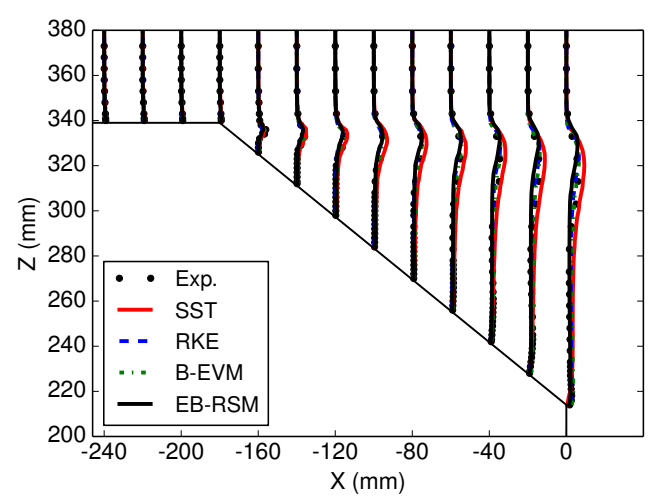

(c)

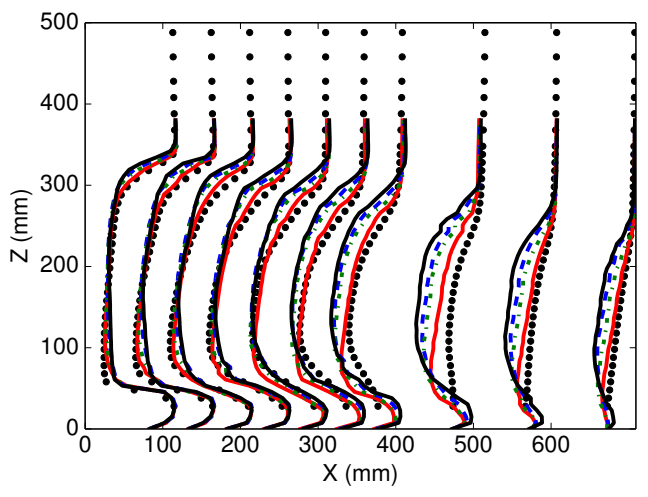

(b)

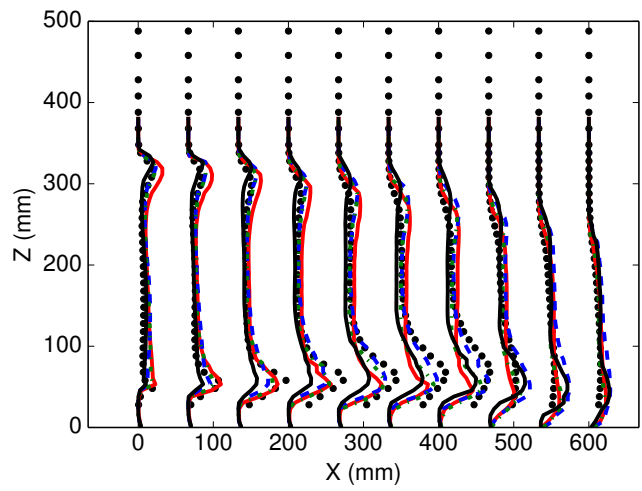

(d)

Figure 6: Streamwise velocity (a) over and at the (b) rear of the Ahmed car body at 35 degrees and Turbulent Kinetic Energy (TKE) (c) over and at the (d) rear of the Ahmed car body at 35 degrees for different RANS models

sults are in agreement again with many previous studies, where unsatisfactory performance was observed with RANS models, both EVM and RSM variants [20,21]. A common theme from all of these studies was an under-prediction of the turbulent kinetic energy in the initial separated shear layer, which is also observed here.

In the work of Ashton et al. [19] a mesh convergence study found that mesh convergence was achieved for all models on the 16 million cell grid which is currently in use, therefore we discount a lack of grid resolution for the poor performance of the RANS models. In order to also eliminate code-related errors, the same mesh and models were tested in the open source finite-volume code, Code_Saturne. Whilst the exact numerics and model implementation may be different between codes, it can be seen in Figure 8 that the models provide similar performance, with all models over-predicting the separation region. We therefore believe that these results are code-independent.

As linear eddy-viscosity and Reynolds stress models give nearly identical results (in both codes as well as other studies), it can also be concluded that it is not purely an issue of models lacking the ability to capture anisotropy or near-wall modelling (although how well they do this may be contributing to the poor performance). Other $\varepsilon$ based RSM models (such as the LRR model [37]) were tested and yielded no improvement. The under prediction of the 


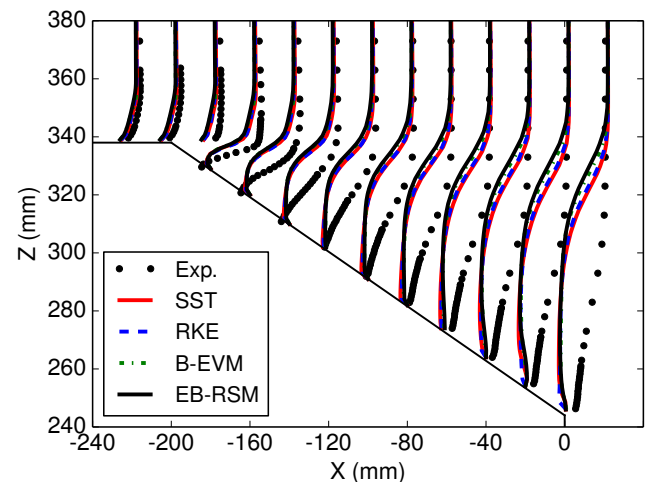

(a)

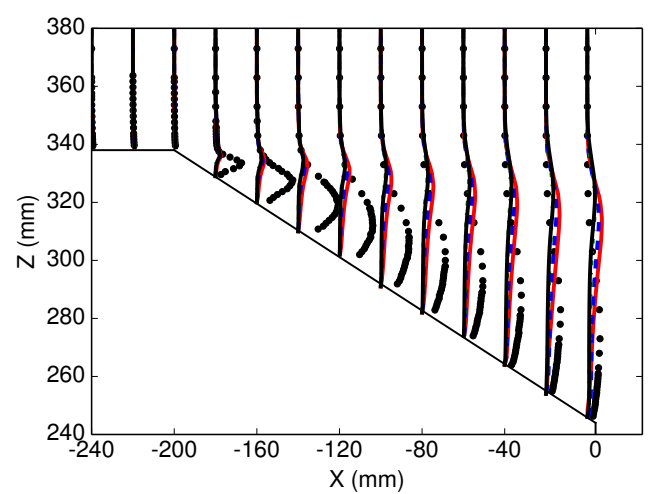

(c)

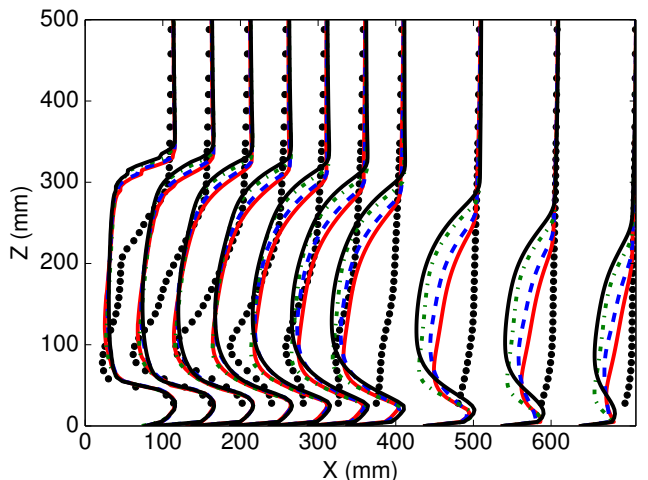

(b)

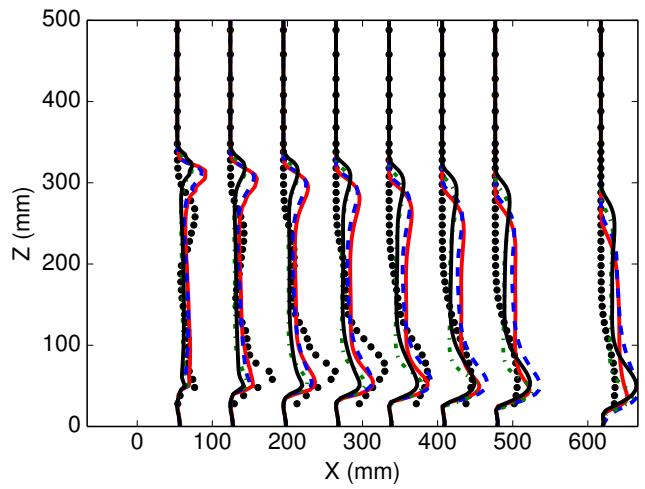

(d)

Figure 7: Streamwise velocity (a) over and at the (b) rear of the Ahmed car body at 25 degrees and Turbulent Kinetic Energy (TKE) (c) over and at the (d) rear of the Ahmed car body at 25 degrees for different RANS models

turbulence in the initial separated shear layer has been investigated in more detail in the work of [19] and this same failure of common RANS models has been found in a number of other separated flows e.g 2D wall-mounted hump \& 2D periodic hills. The authors believe this is an area of potential development for future RANS models, and work is currently underway by the authors to investigate this in the spirit of work done by Rumsey et al. [38] on sensitising models to increase the turbulence in this initial separated shear layer.

\subsubsection{DrivAer model: RANS}

The same RANS models were applied to the DrivAer case (with the inclusion of the Spalart Allmaras model) which now represents a realistic car geometry. The results of these for the lift and drag coefficients are summarized in Table 5 and Figures $9 \& 10$ are compared to the experimental data and simulations from other research groups. The results from this study are all using the fine mesh ( 80 million cells), although it was seen that there was very little difference between the medium and fine grids for most models, as shown in Figure 11.

It can be seen firstly that none of the models can correctly predict all the car configurations. Whilst one model may 


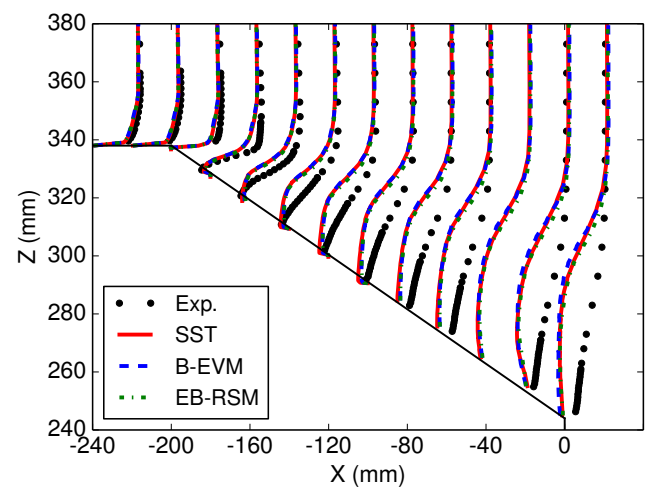

(a)

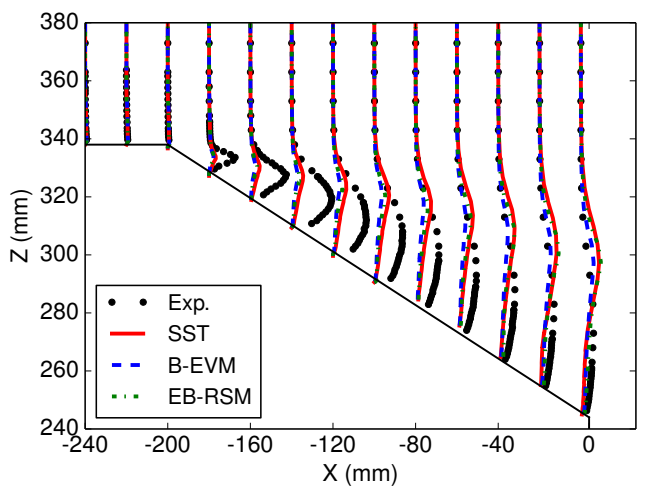

(b)

Figure 8: (a) Streamwise velocity and (b) Turbulent Kinetic Energy for different RANS models at $y=0$ for the Ahmed car body at 25 degrees with Code Saturne

perform predict the drag force correctly for one configuration (SST for Fastback), it is unable to predict the correct drag force for the other car configuration and has a significant error with the lift coefficient. This overprediction of the lift force is in agreement with the other studies, which each used different meshing strategies and resolution. An inspection of the drag contribution from each part of the car showed that the majority of the differences between the models occurred in the rear of the car, which is not surprising as this is the area of greatest flow separation. There is a noticeable trend towards the RANS models predicting the fastback configuration more accurately, which has no separation over the rear window compared to the estate configuration. Whilst certain RANS models predict the drag force better than others (e.g SST compared to EBRSM), this appears to be due to an over-prediction of the lift force, which in turn produces an induced drag component. None of the RANS models captures the correct lift-to-drag ratio for either car configuration.

Table 5 also provides further insight by showing the difference in lift and drag coefficient between the estate and fastback configurations. Whilst all the models capture the trend of a reduction in drag coefficient, they all fail to capture the magnitude. Typically for industrial automotive flows it is accepted that RANS models cannot capture the absolute force coefficients, so instead they are used to assess the magnitude and direction of a trend. Looking at geometry trends removes constant sources of error, which can be useful for such a complex geometry however such high errors suggest the models are missing some fundamental flow features between the two configuration (i.e the extent of the separation region).

The pressure coefficient over the top of both the fastback and estate vehicles is shown in Figure 12. Inspection of Figure 12(a) for the fastback vehicle, shows that each model exhibits broadly similar pressure distributions apart from the region at bottom of the front window/bonnet intersection. Here the SST (in agreement with the results of Heft et al. [4]) predicts too strong suction. All models under-predict the pressure coefficient (for both the fastback and estate vehicles) over the roof of the car, which is also seen in Heft et al. [4]. This is likely due to the lack of a 
strut in the CFD model, which connects the car model to the wind tunnel. The flow over the roof of the car should be attached and therefore simple to capture with any turbulence model. Without also having the precise geometry of the wind tunnel, it is not possible to match the same blockage conditions. For the estate configuration in Figure 12(b), there is clear split between the models over the rear portion of the car. This is due to differences in the model ability to capture the flow separation, which is also reflected in the drag coefficient (SST having the largest drag for greater flow separation).

The previous figures have shown the $C_{p}$ along a single plane at the centreline, Figure 13 however provides us with the pressure coefficient over the rear window of the car for the all the models for both configurations. Here it can be seen that the fastback is predicted more accurately by all the turbulence models (which is also reflected in the drag coefficient), with relatively little difference between them. For the estate configuration, the pressure coefficient is far from the experimental values (which is again reflected in the drag coefficient).

Figures $14 \& 15$ shows a visualization of the separated regions of the flow (i.e $U_{i}<0$ ) for the SST, EBRSM and the SST-IDDES model (to be discussed in the next section). It can seen that there is a noticeable difference in the shape of the wake for the both the fastback and estate configurations. These difference is likely to be one of the major contributions to the difference observed in the lift and drag forces.

Figures 16-19 show the mean streamwise velocity and modelled \& resolved TKE for selected slices along and behind the car body. Whilst these are only selected slices, they show different wake patterns behind the two example RANS models (SST \& EBRSM) although the difference is relatively small at these positions. The major observation from these figures is the under-prediction of TKE compared to the hybrid RANS-LES solutions. Although there is no experimental data to compare against this under-prediction of the TKE relative to the hybrid RANS-LES results, this agrees closely with the Ahmed car body at $25^{\circ}$, and suggests a common source of error from the RANS models.

This inability to correctly predict the flow regardless of the turbulence model, mesh (mesh refinement was evaluated up to 80 million cells) was a major motivation to test the hybrid RANS-LES models in this paper. Whilst it cannot be said that no RANS model could ever predict the flow correctly, the current state of the art models have not showed acceptable performance both for the Ahmed car body and this realistic car model.

\subsection{Hybrid RANS-LES}

It has been shown for both the Ahmed car body and DrivAer vehicle that no RANS model is capable of predicting the flow correctly for all configurations. This suggests that whilst they may offer good predictive capability for particular flow types, they are unable to consistently provide good enough accuracy (i.e within 5\% of absolute loads). For this reason we evaluate hybrid RANS-LES models, in particular Detached Eddy Simulation initially for the Ahmed car body (at the more challenging $25^{\circ}$ degrees) and then later the DrivAer realistic car geometry. These type of methods offer close to the theoretical predictive capability of LES models but with much reduced computational resources. 


\begin{tabular}{|c|c|c|c|c|c|c|}
\hline \multirow{2}{*}{ Model } & \multicolumn{2}{|c|}{ Estate } & \multicolumn{2}{c|}{ Fastback } & \multicolumn{2}{c|}{ Vehicle Delta } \\
\cline { 2 - 7 } & $C_{D}$ & $C_{L}$ & $C_{D}$ & $C_{L}$ & $C_{D}$ & $C_{L}$ \\
\hline Exp. & $\mathbf{0 . 2 9 4}$ & $\mathbf{- 0 . 1 2}$ & $\mathbf{0 . 2 6 1}$ & $\mathbf{0 . 0 1}$ & $\mathbf{0 . 0 3 3}$ & $\mathbf{0 . 1 3}$ \\
\hline$k-\omega$ SST from Heft et al. [3] & $\mathrm{n} / \mathrm{a}$ & $\mathrm{n} / \mathrm{a}$ & 0.243 & $\mathrm{n} / \mathrm{a}$ & $\mathrm{n} / \mathrm{a}$ & $\mathrm{n} / \mathrm{a}$ \\
$k-\omega$ SST from Peters et al. [35] & $\mathrm{n} / \mathrm{a}$ & $\mathrm{n} / \mathrm{a}$ & 0.256 & 0.069 & $\mathrm{n} / \mathrm{a}$ & $\mathrm{n} / \mathrm{a}$ \\
EARSM from Guilmineau [36] & $\mathrm{n} / \mathrm{a}$ & $\mathrm{n} / \mathrm{a}$ & 0.254 & 0.079 & $\mathrm{n} / \mathrm{a}$ & $\mathrm{n} / \mathrm{a}$ \\
\hline Spalart Allmaras (SA) & 0.280 & 0.054 & 0.260 & 0.136 & 0.02 & 0.084 \\
Realizable $k-\varepsilon$ (RKE) & 0.260 & -0.026 & 0.244 & 0.085 & 0.016 & 0.11 \\
$k-\omega$ SST & 0.275 & 0.0436 & 0.260 & 0.124 & 0.00 & 0.08 \\
$k-\varepsilon$ B-EVM & 0.253 & 0.007 & 0.2435 & 0.116 & 0.0095 & 0.109 \\
EB-RSM & 0.256 & -0.029 & 0.2482 & 0.075 & 0.0078 & 0.104 \\
\hline SST-DES from Guilmineau [36] & $\mathrm{n} / \mathrm{a}$ & $\mathrm{n} / \mathrm{a}$ & 0.266 & 0.024 & $\mathrm{n} / \mathrm{a}$ & $\mathrm{n} / \mathrm{a}$ \\
\hline SST IDDES (Coarse) & 0.310 & -0.096 & 0.268 & 0.011 & 0.042 & 0.107 \\
SST IDDES (Fine) & 0.307 & -0.131 & 0.2615 & 0.024 & 0.0455 & 0.155 \\
SA IDDES (Fine) & 0.313 & -0.136 & $\mathrm{n} / \mathrm{a}$ & $\mathrm{n} / \mathrm{a}$ & $\mathrm{n} / \mathrm{a}$ & $\mathrm{n} / \mathrm{a}$ \\
SA DDES (Fine) & 0.307 & -0.13 & $\mathrm{n} / \mathrm{a}$ & $\mathrm{n} / \mathrm{a}$ & $\mathrm{n} / \mathrm{a}$ & $\mathrm{n} / \mathrm{a}$ \\
\hline
\end{tabular}

Table 5: Lift and drag coefficients for the computational and experimental results for the fastback and estate configurations of the DrivAer using different RANS models on 80 million fine RANS mesh and a range of DES variants on the coarse and fine meshes 


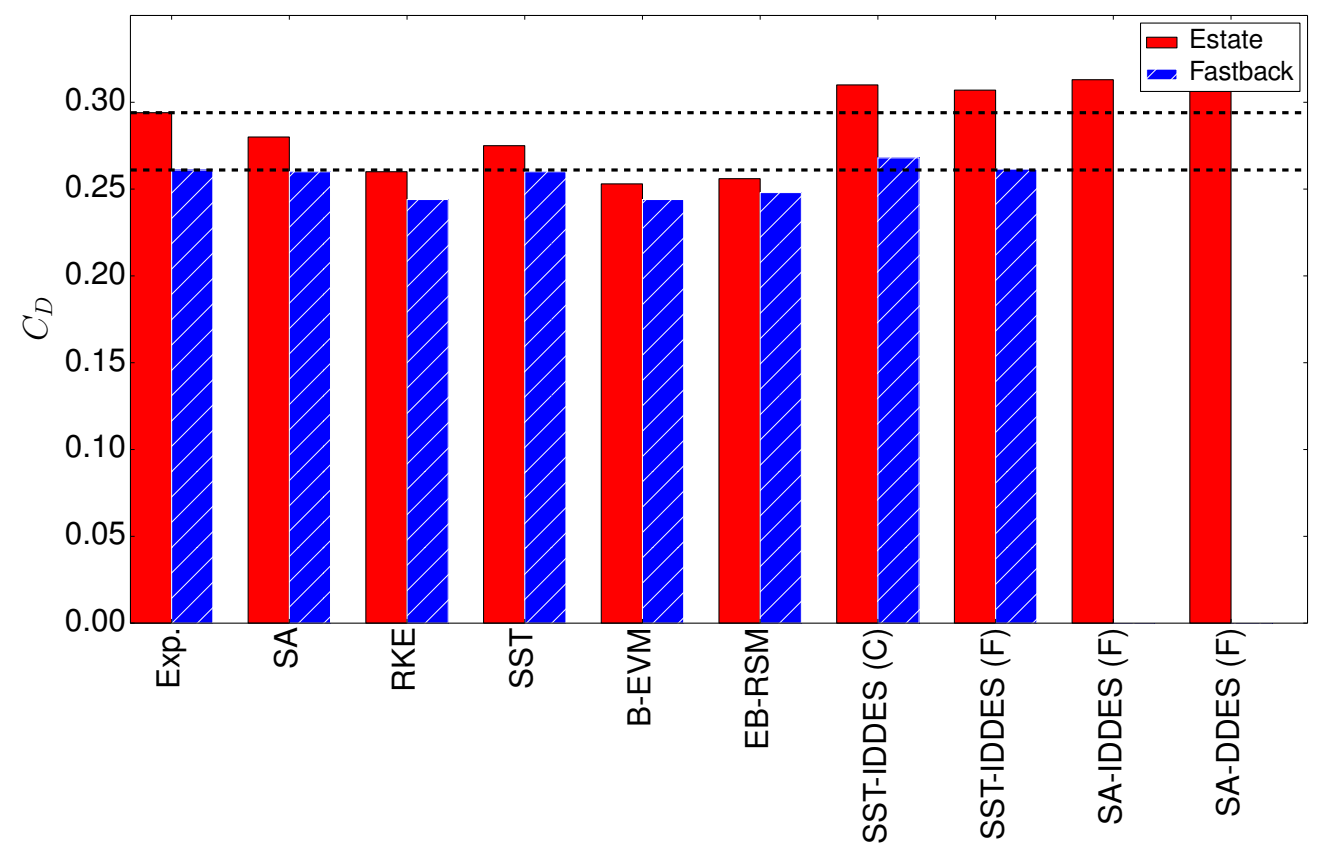

Figure 9: Drag Coefficient for the estate and fastback configurations using RANS and DES models

\subsubsection{Ahmed car body: DES}

Figure 20 shows the mean streamwise velocity and turbulent kinetic energy over and behind the Ahmed car body using the SST, EBRSM and SST-IDDES models. It can be seen that the SST-IDDES model provides an improved prediction of the streamwise velocity as a result of predicting a large TKE in the initial separated region. There is still however a clear under-prediction of the TKE in the initial separated shear layer which means too little turbulent mixing and an over-prediction of the separation region. This is visualized more clearly in Figure 21 which shows the regions of separated flow for the SST, EBRSM and SST-IDDES models, it's clear that the RANS model predict a much larger recirculation region compared to the SST-IDDES model. To assess if this can be improved by either ulitizing a different underlying RANS models or a different DES variant, Figure 22 shows the results for the SA IDDES and SA-DDES models. It can be seen that there is very little sensitivity to either the DES variant or underlying RANS models for this case, with none improving the underprediction of TKE in the initial separated shear layer region.

To establish that any errors do not arise from the use of STAR-CCM+ (i.e the codes numerics or implementation), the same case and mesh was run in Code_ Saturne using the SST-DDES model (as shown in Figure 22(c)). It can be seen that there is little or no difference between this and the all the DES variants run in STAR-CCM+. This is further seen through the visualization of iso-contours in Figure 23, where there is little difference in the structures observed.

For all the DES variants, there is a clear improvement over the RANS models in the prediction of both the turbulent kinetic energy and the streamwise velocity. However, there is still an underprediction of the turbulence in the initial separated shear layer, as shown in Figure 22(c). This exists no matter the grid refinement (up to 16 million cells [19], 


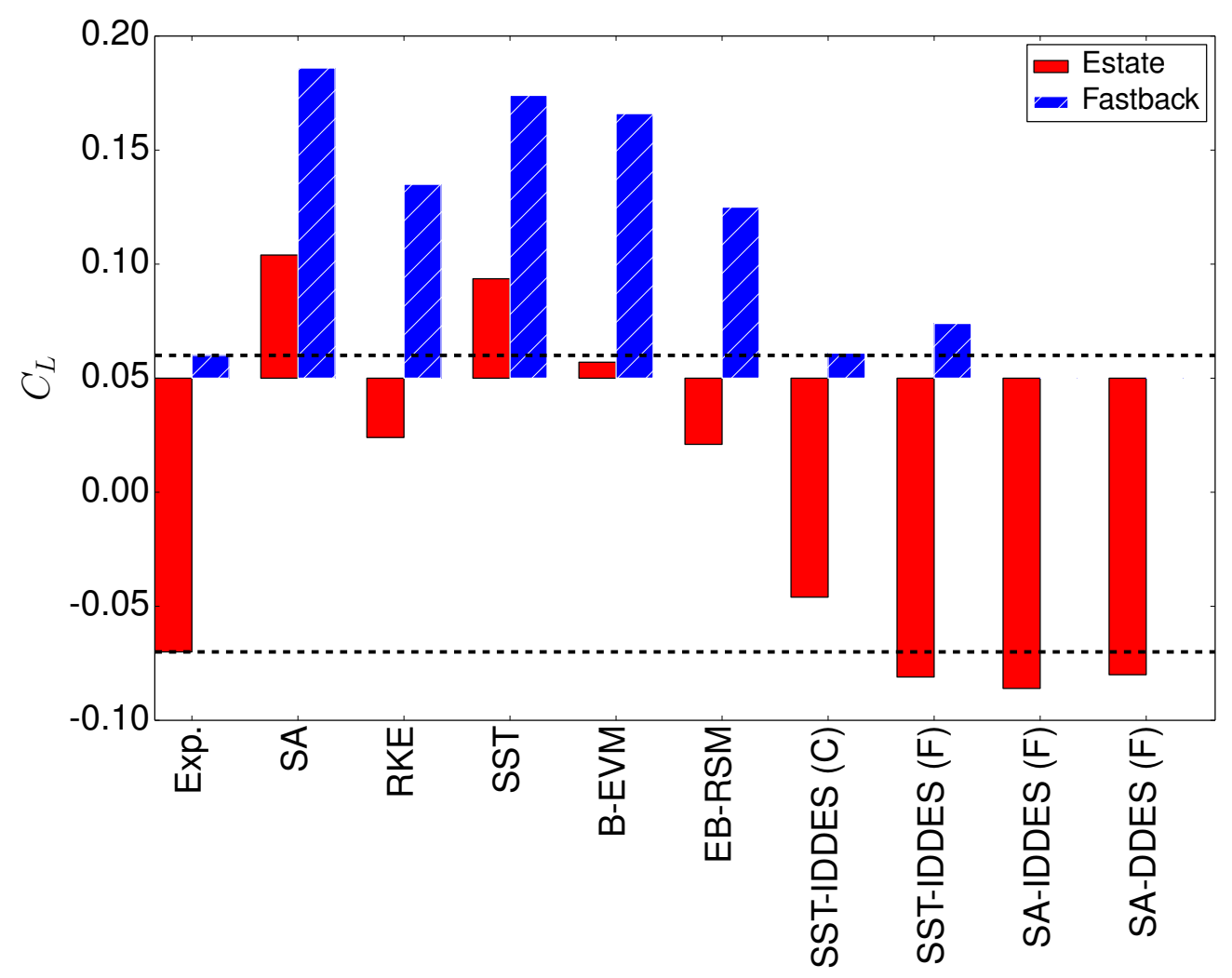

Figure 10: Lift coefficient for the estate and fastback configurations using RANS and DES models

DES model formulation (IDDES, DDES) nor the code itself (STAR-CCM+, Code Saturne). Further analysis of this case can be found in Ashton et al. [19], including more detailed analysis of the failure of the RANS and DES variants.

\subsubsection{DrivAer Model: DES}

It was seen that using hybrid RANS-LES for the Ahmed car body, provided an improvement over all RANS models in terms of a more accurate prediction of the separation region (although still not in complete agreement with experimental data). As the conclusions from the RANS study of both the Ahmed car body and DrivAer were similar, we now apply hybrid RANS-LES methods to the DrivAer vehicle to assess if these also provide an improvement.

We evaluate two meshes for the hybrid RANS-LES calculations, a coarse 80 million cell grid composed of prism cells in the boundary layer and polyhedral cells away from the wall, this is the same as the fine grid used in the RANS simulations. Secondly we use a mesh of 100 million cells, with now hexahedral cells away from the wall to provide a more structured cell layout. A third finer mesh was evaluated but insufficient time-sampling has taken place (initial results show little difference between this and the 100 million cell grid). These meshes are summarized in Table 4 .

The results for the force coefficients are shown in Table 5 and Figures $9 \& 10$ for the fastback and estate configurations, as well as the pressure coefficient in Figure 24. The results firstly show that all hybrid RANS-LES results 


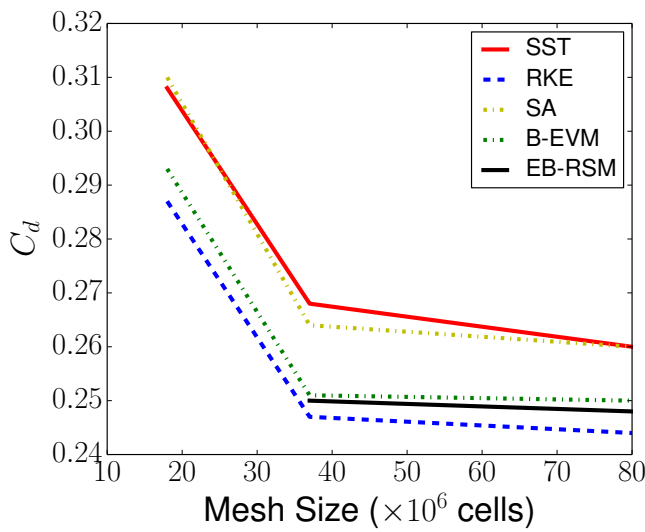

(a)

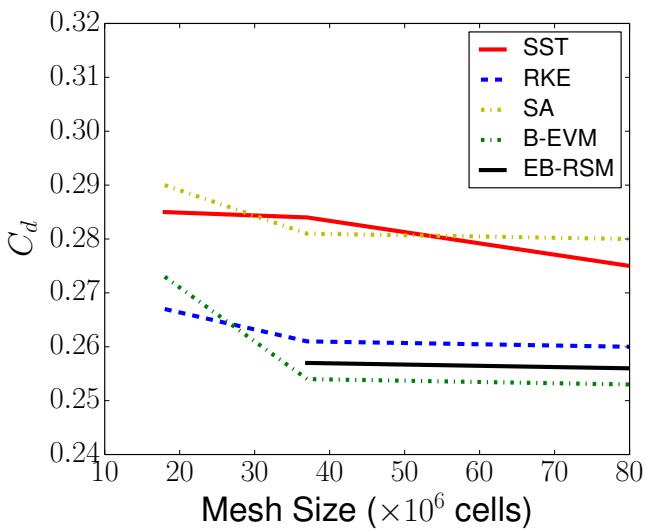

(b)

Figure 11: Mesh Refinement for the fastback \& estate configuration using RANS models

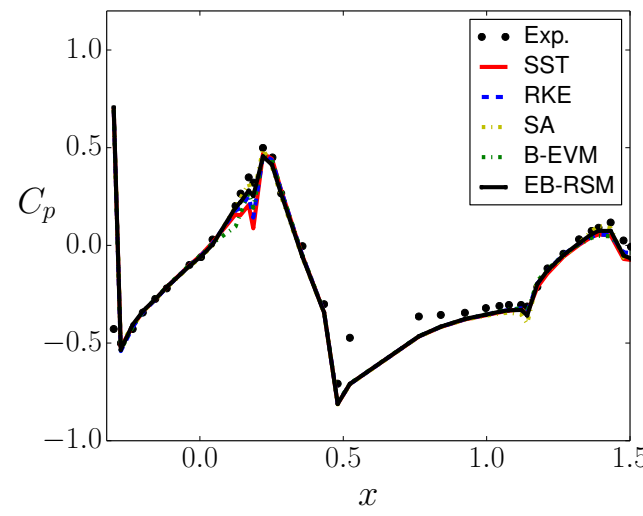

(a)

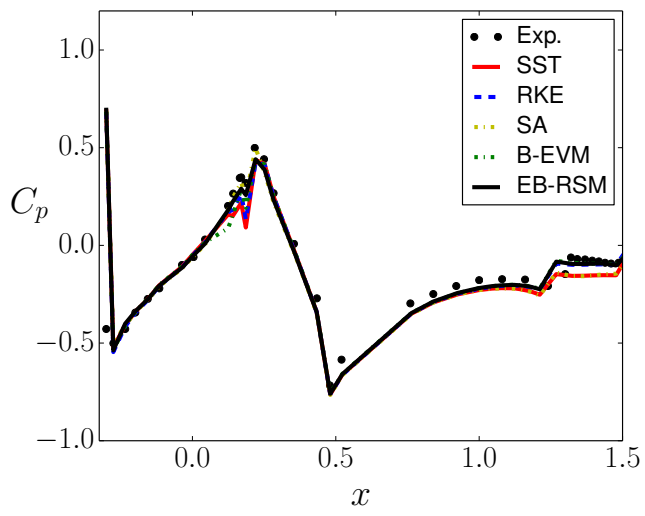

(b)

Figure 12: (a) Pressure Coefficient over the top of the Fastback configuration for each RANS model. (b) Pressure Coefficient over the top of the Estate configuration for each RANS model.

(regardless of mesh resolution or DES variant) provide a better prediction of the flow for both vehicle configurations. This is most noticeable for the lift coefficient, which was poorly predicted by the RANS models, but predicted relatively well for all the hybrid RANS-LES models. Interestingly, in a similar fashion to the Ahmed car body, there is relatively little difference between the DES variants (SA IDDES, DDES, SST-IDDES) for this flow, although this might be more pronounced with a finer mesh and/or the addition of inflow turbulence.There is a noticeable improvement with the fine mesh for the SST-IDDES computations, which suggests that the polyhedral mesh has additional numerical dissipation (as there is only 20 million cells difference between coarse and fine meshes).

Table 5 also provides the change in drag coefficient between the Estate and Fastback configurations. Compared to the RANS results it can be seen that not only is the direction of the trend correct but the magnitude is much closer with the hybrid RANS-LES models which combined with the absolute values, makes the hybrid RANS-LES much 


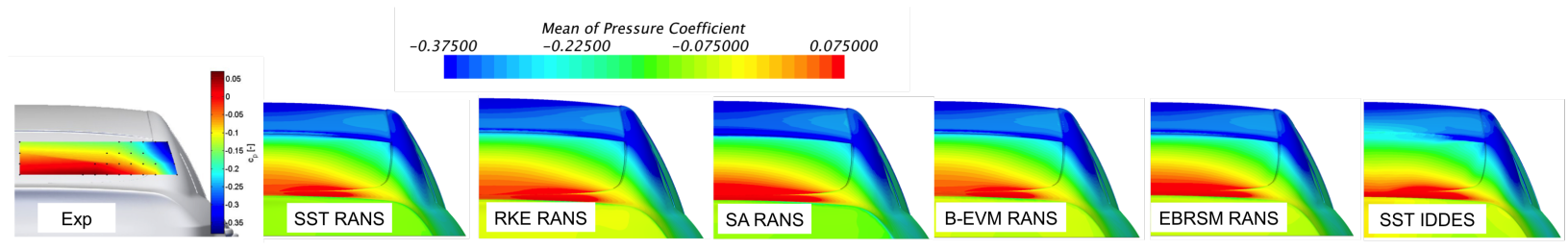

(a)

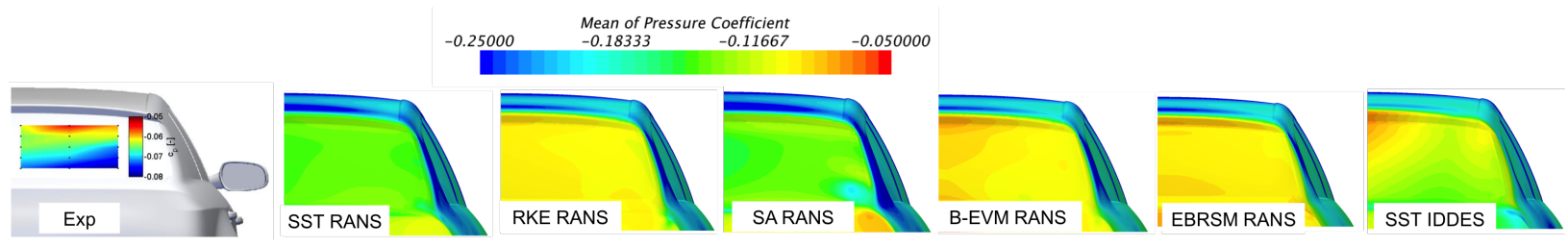

(b)

Figure 13: Mean Pressure Coefficient distribution for the (a) Fastback and (b) Estate vehicles for Experimental data and RANS CFD simulations

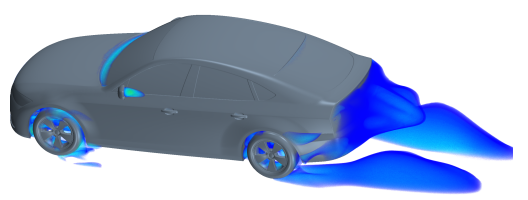

(a)

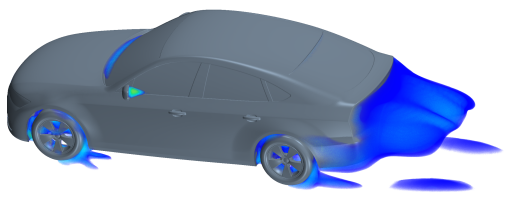

(b)

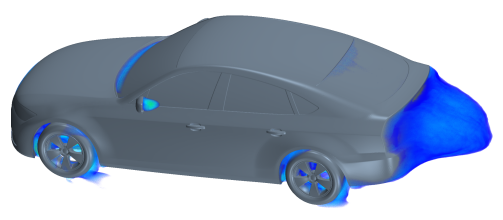

(c)

Figure 14: Volume render, showing separation regions for (a) SST RANS, (b) EBRSM RANS and (c) SST-IDDES model for fastback vehicle

more accurate overall than any of the RANS models. These differences can be seen in the wake structures in Figures $14 \& 15$ for the fastback and estate configurations respectively. In a similar fashion to the Ahmed car body, the RANS models predict a larger recirculation region and a different wake structure than the hybrid RANS-LES models. Whilst there is no experimental data for the 3D wake, the close agreement with both the lift and drag for the hybrid RANS-LES models suggests this is a more accurate representation of the wake structures.

Figure 13 also shows the $C_{P}$ distribution over the rear window of the Estate and Fastback configurations against the RANS and experimental data [4]. For the fastback configuration, both RANS and DES methods predict a similar

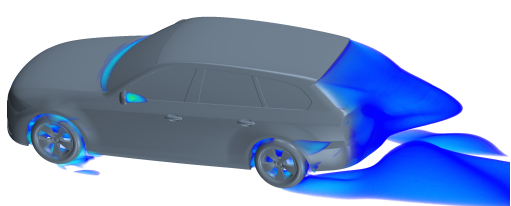

(a)

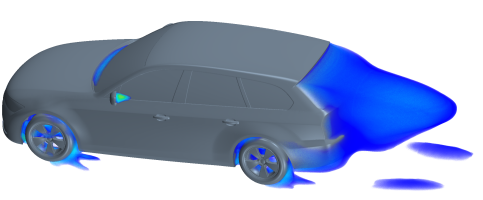

(b)

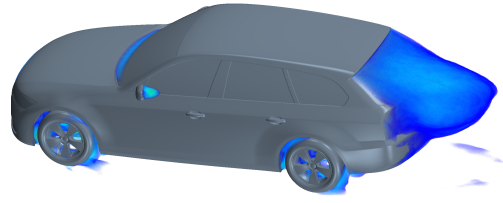

(c)

Figure 15: Volume render, showing separation regions for (a) SST RANS, (b) EBRSM RANS and (c) SST-IDDES model for estate vehicle 


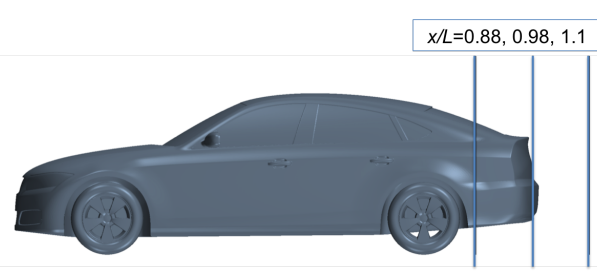

(a)

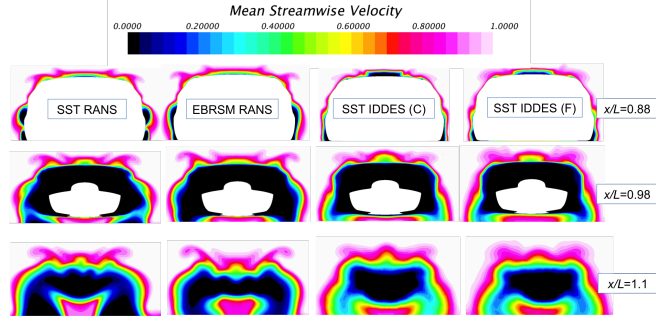

(b)

Figure 16: Mean Streamwise velocity at selected slices of the Fastback vehicle for the SST \& EBRSM RANS models and the SST-IDDES model on the coarse and fine mesh

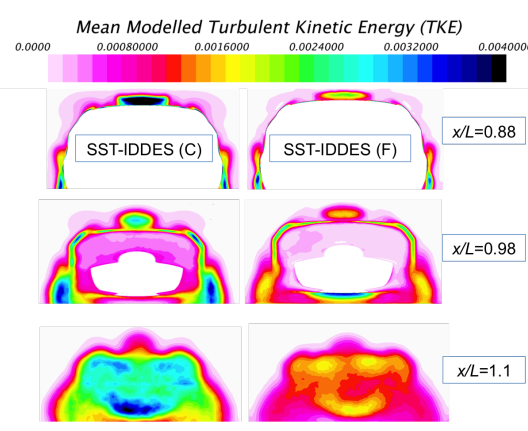

(a)

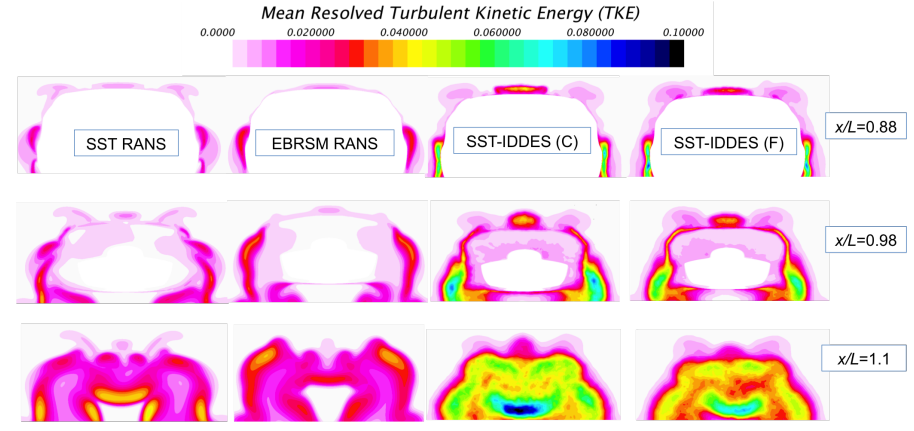

(b)

Figure 17: (a) Mean Modelled Turbulent Kinetic Energy (TKE) \& (b) Mean Resolved Turbulent Kinetic Energy (TKE) at selected slices of the Fastback vehicle for the SST \& EBRSM RANS models and the SST-IDDES model on the coarse and fine mesh

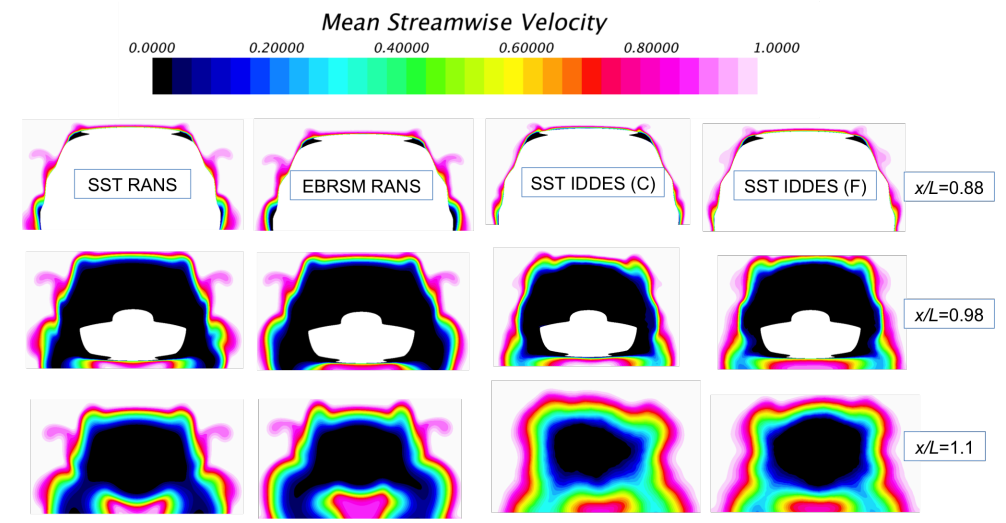

Figure 18: Mean Streamwise Velocity at selected slices of the Estate vehicle for the SST \& EBRSM RANS models and the SST-IDDES model on the coarse and fine mesh 


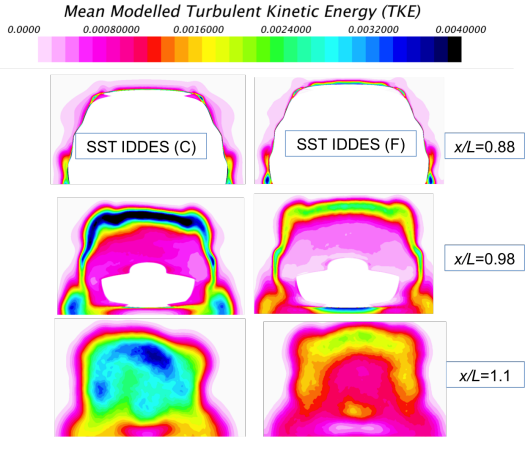

(a)

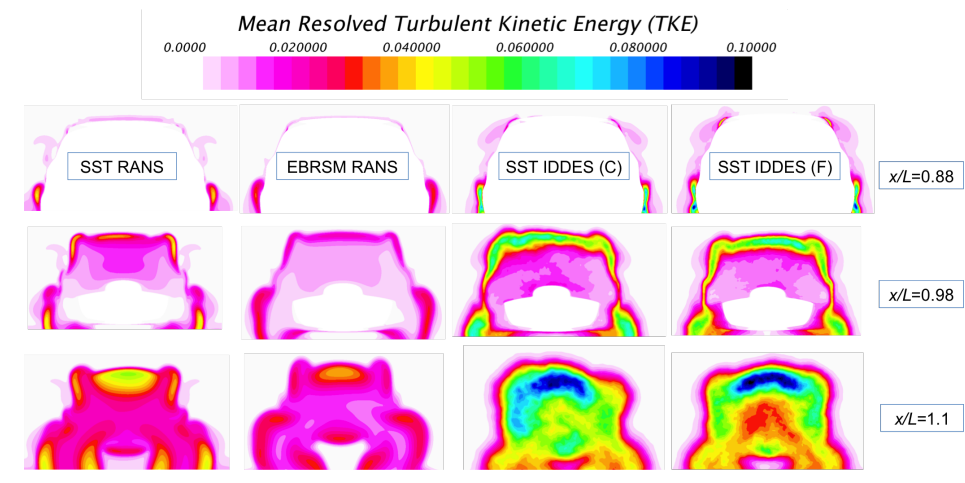

(b)

Figure 19: (a) Mean Modelled Turbulent Kinetic Energy (TKE) \& (b) Mean Resolved Turbulent Kinetic Energy (TKE) at selected slices of the Estate vehicle for the SST \& EBRSM RANS models and the SST-IDDES model on the coarse and fine mesh

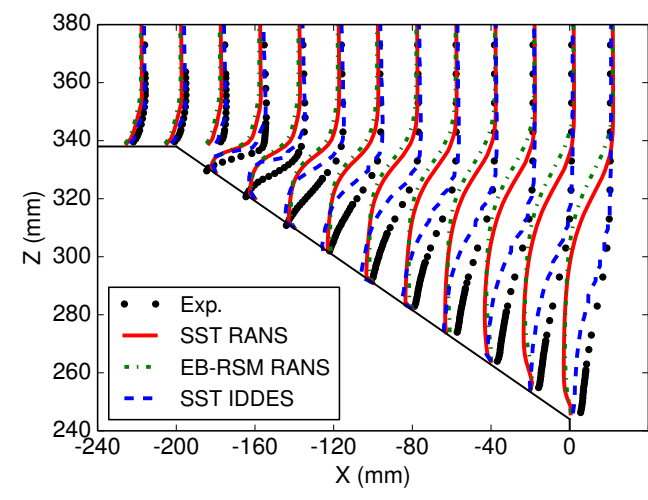

(a)

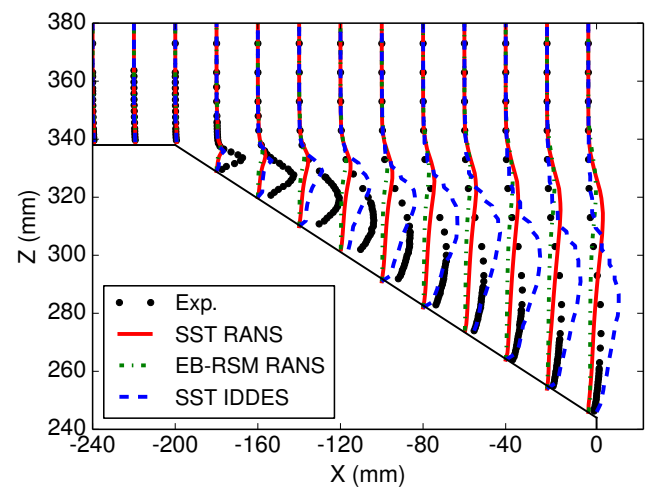

(c)

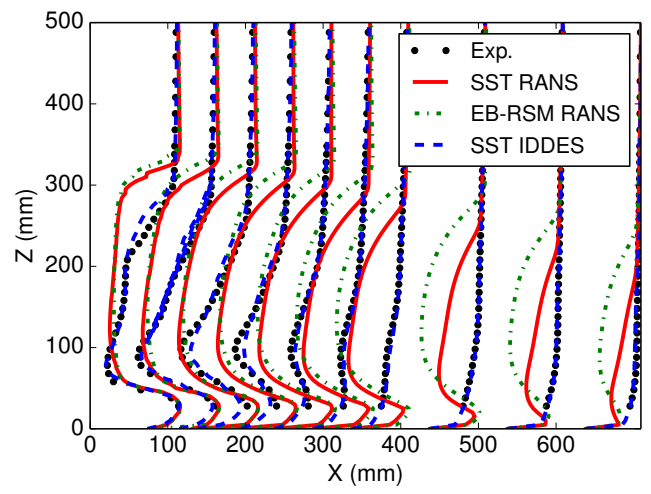

(b)

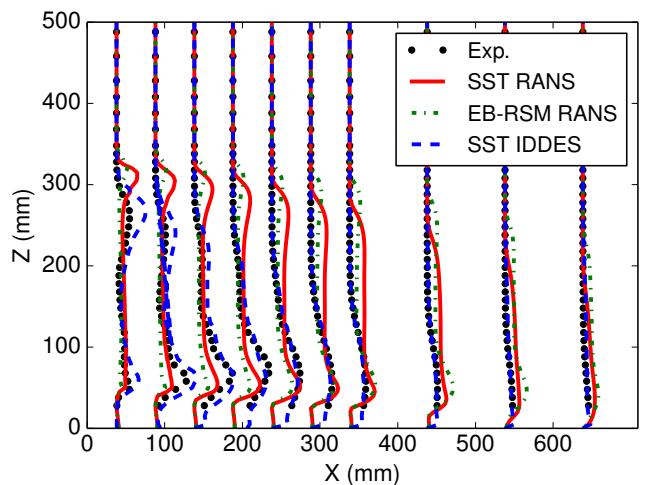

(d)

Figure 20: (a) Mean Streamwise velocity along and (b) behind the Ahmed car body and (c) Mean turbulent kinetic energy along and (d) behind the Ahmed car body for the SST \& EBRSM RANS and SST-IDDES models 


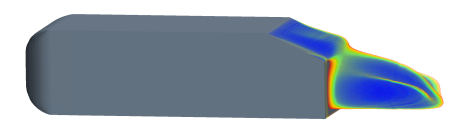

(a)

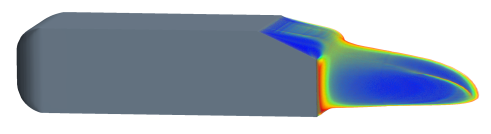

(b)

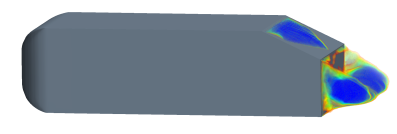

(c)

Figure 21: Visualization of the separated flow for the Ahmed car body using the (a) SST RANS, (b) EBRSM RANS and (c) the SST-IDDES model

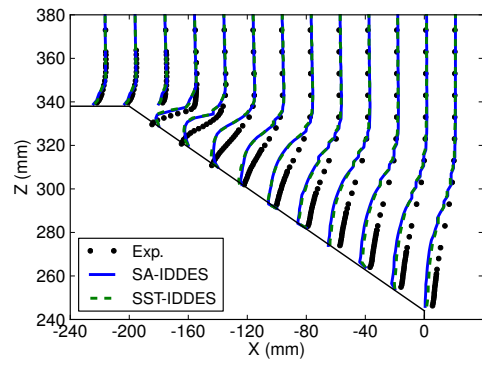

(a)

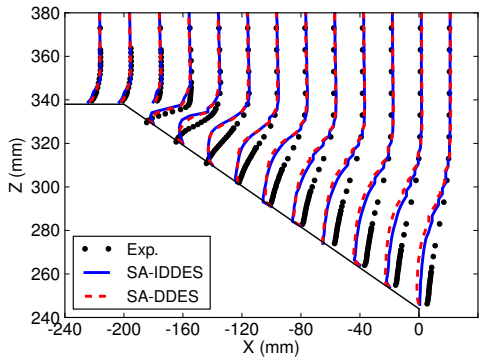

(b)

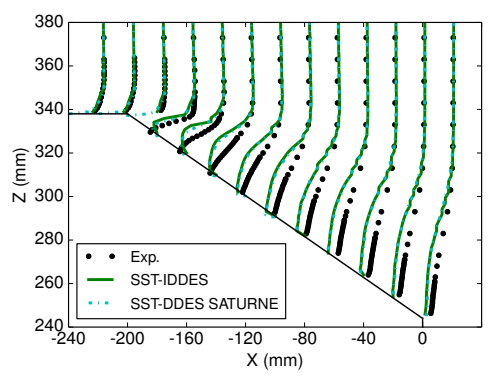

(c)

Figure 22: Mean Streamwise velocity along the Ahmed car body for the (a) SA-IDDES and SST-IDDES models, (b) SA-IDDES and SA-DDES models and (c) SST-IDDES and SST-DDES (Code Saturne) models.

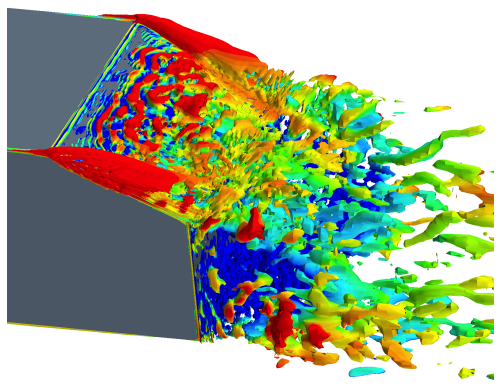

(a)

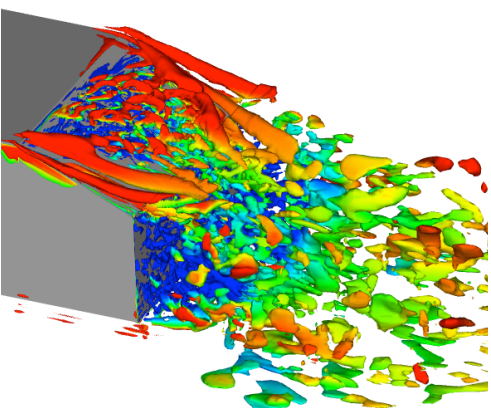

(b)

Figure 23: (a) Iso surface contours of the $\lambda_{2}$ function $\left(\lambda_{2}=200\right)$ for the Ahmed car body using the SST-IDDES model, (b) the SST-DDES model in Code Saturne 
distribution to the experimental data, however both slightly under-predict the pressure recovery. For the Estate configuration neither the RANS and DES methods can capture the correct distribution. Note that the scaling has been changed to make the differences between RANS and DES more visible. There may however be differences due to the exact wind tunnel domain not being replicated in the CFD models which could affect the pressure coefficient. Both DES and RANS models over-predict the negative pressure on the rear, which indicates too large separation prediction. Unfortunately there is no skin-friction or oil flow data from the experiment but it is clear the estate configuration is most challenging. The DES models provide better agreement than the RANS which is reflected somewhat in the drag coefficient.

We can see from all the previous figures that firstly no RANS model can correctly predict the $C_{D}$ or $C_{p}$ for any configuration and that whilst the DES methods give a better prediction they still cannot predict the correct flow, particularly for the estate configuration. It is important to try to ascertain how the DES models provide this improved accuracy.

\section{Turbulence levels}

Figure 17 shows both the modeled and resolved TKE for the fastback vehicle. Whilst there is no experimental data to compare to, we can see that firstly the modeled contribution is small (5\%) in comparison to the resolved content suggesting that the models are operating in LES mode at these locations. This does not however tell us whether the resolved turbulence levels are high enough. For the Ahmed body it was found that all RANS models under-predicted the level of turbulence in the initial separated shear layer and that also even DES variants on a fine mesh still underpredicted the values compared to the experimental data [19]. In Figure 17(b), the level of TKE predicted by the SST-RANS model can be compared to the resolved TKE from the DES results. In a similar fashion to the Ahmed car body it can be seen that the RANS model predicts a lower value of TKE than any of the DES variants. This helps to explain why the RANS models exhibits greater flow separation in Figure 16(b), as reduced levels of TKE would result in lower levels of turbulent mixing and a greater recirculation region.

Figures $18 \& 19$ show the mean streamwise velocity, modeled TKE and resolved TKE for the estate configuration for different DES variants and the SST-RANS model. A similar conclusion to the fastback can be observed where the RANS model predicts a lower level of TKE and as a consequence shows a larger recirculation region than any of the DES variants.

Whilst it has been established that the DES variants provides better accuracy on both car configurations than RANS models, they still exhibit errors with the lift \& drag coefficients and also for the Cp over the rear window. So far it has been shown that the relative RANS-DES performance for the DrivAer cars appear to match the traits from the Ahmed car body (in terms of TKE and recirculation size), thus it is therefore likely that the level of TKE is still under-predicted in the initial separated shear layer which results in too large recirculation length. 


\subsection{Embedded LES}

The solution for the Ahmed car body (as detailed in Ashton et al. [19]) was to inject synthetic turbulence (via the DFSEM approach [39]) in a one-way coupled embedded DES region, thereby over-coming the lack of TKE in the initial separated shear layer. This provided the unsteady velocity fluctuations prior to the separation point, which allowed the flow to move into a fully resolved state in the initial separated shear layer. This showed very promising performance for the Ahmed car body (as shown in Figure 25) and provided much closer prediction of the turbulence in the initial separated shear layer. As this was a one-way coupled approach, it meant cutting the domain and car prior to the separation point and placing an inlet boundary condition on this plane, at which synthetic turbulence could be produced.

Unfortunately for a realistic configuration, like the DrivAer model, this approach would not be suitable. Cutting the car in half firstly poses a challenge for the modelling of the vortices that move down the car, which may be disturbed by such an approach and secondly there is an obvious coupling effect of the rear separation on the whole circulation of the car, thus only a two-way coupled approach would be suitable (which is also strictly true for the Ahmed car body). The authors are currently working on developing such an approach but at present this was not available to evaluate. Nevertheless it is of the opinion of the authors that injecting synthetic turbulence prior to the separation point would help overcome the current shortcomings of the DES approach, in a similar fashion to the Ahmed car body, and hopefully provide an even closer agreement to the experimental data.

\subsection{Computational Expense}

A major consideration for industrial users of CFD is not only the accuracy of the method but also the computational cost. Whilst it has shown that hybrid RANS-LES methods, in particular DES provides a clear advantage over RANS models for the two test-cases evaluated, it is important to also assess the computational cost of each approach. For this, shown in Table 6, we compare a RANS simulation using the SST model on the DrivAer fastback configuration to the SST-IDDES model used also on the DrivAer fastback configuration. A constant number of cells per core is used to decide upon the total number of cores used; 144,000 cells per core in this case. This represents the scalability possible with the majority of industrial CFD codes.

The RANS simulations were run steady-state so the number of iterations required to reach the convergence criteria is stated. For the SST-IDDES simulation a dual-time stepping transient approach is used, thus the time per iteration is now the time per time step (of which 5 inner iterations are used). We calculate a relative cost based upon the additional compute time as well as the additional computational expense of using more HPC resources. It can be seen that the hybrid RANS-LES simulation is 17 times more expensive than the RANS computation. Whilst these values could be increased or decreased depending on specific optimzation of the mesh or solution strategy this difference explains why RANS models are still the workhorse of many industries. Nevertheless, with the inaccuracies highlighted in this paper of the current state-of-the-art RANS models, the additional computational time should now be deemed necessary. 
Whilst the current turnaround time is 2 days (on 704 cores), increased scalability of CFD codes and greater access to HPC resources could bring this under 24 hours, as highlighted in Table 6 when using 2048 cores on MareNostrum HPC facility at Barcelona Supercomputing Centre (BSC). On the other hand, improved RANS models capable of providing accuracy closer to hybrid RANS-LES would become very attractive and thus it is the opinion of the authors that there is a still a real need for further research to produce improved RANS models, particularly for complex separated flows.

Table 6: Computational expense of each RANS and DES simulation for the DrivAer vehicle

\begin{tabular}{|c|c|c|c|c|c|c|}
\hline Method & Cells & Cores $^{1}$ & Temporal Scheme & Time per iteration & Compute time & Relative cost $^{2}$ \\
\hline SST RANS & $80 \times 10^{6}$ & 512 & Steady & $6 s(2500$ it. $)$ & $4 \mathrm{hrs}$ & 1 \\
SST IDDES & $100 \times 10^{6}$ & 704 & Transient $\left(5 \times 10^{-5}\right)$ & $7 \mathrm{~s}(26,000 \mathrm{t}$. steps $)$ & $50 \mathrm{hrs}$ & 17 \\
\hline SST IDDES & $100 \times 10^{6}$ & $2048^{3}$ & Transient $\left(5 \times 10^{-5}\right)$ & $2.5 \mathrm{~s}(26,000 \mathrm{t}$. steps $)$ & $18 \mathrm{hrs}$ & 17 \\
\hline
\end{tabular}

\section{Conclusions}

A comprehensive investigation of RANS and DES models for the Ahmed car body and a realistic automotive model has been conducted. A variety of RANS models, from the one-equation Spalart Allmaras model to a lowReynolds number Reynolds Stress model have shown an inability to consistently correctly capture the flow for both these cases with the under-prediction of the turbulence in the initial separated shear layer a key deficiency. It has been shown that the use of a hybrid RANS-LES model (in this case, Detached Eddy Simulation) offers an advantage over RANS models in terms of the force coefficients, and general flow field for both the Ahmed car body and the DrivAer model. However, for both cases even at the finest mesh level they could not correctly capture the flow. The use of an embedded LES approach with the injection of synthetic turbulence is discussed, and results from using such an approach on the Ahmed car body is briefly presented, however it is noted that further work is required to make this suitable for a complete vehicle simluation. Finally the computational expense of each approach is discussed, showing that hybrid RANS-LES methods are still many times more expensive than steady RANS, which suggests that further work to improve RANS modelling is still an important area, largely because of the potential cost benefit.

\footnotetext{
${ }^{1}$ Using 2.6 GHz Intel Sandy Bridge processors (16 cores per node with 64GB memory), with Mellanox QDR Infiniband

${ }^{2}$ Relative cost calculated as additional compute time $\times$ additional cost of HPC resource (e.g number of cores used)

${ }^{3}$ Performed on MareNostrum at Barcelona Supercomputing Centre (BSC) using 2.6 GHz Intel Sandy Bridge Xeon processors (16 cores per node with 32GB memory), with Mellanox FDR Infiniband
} 


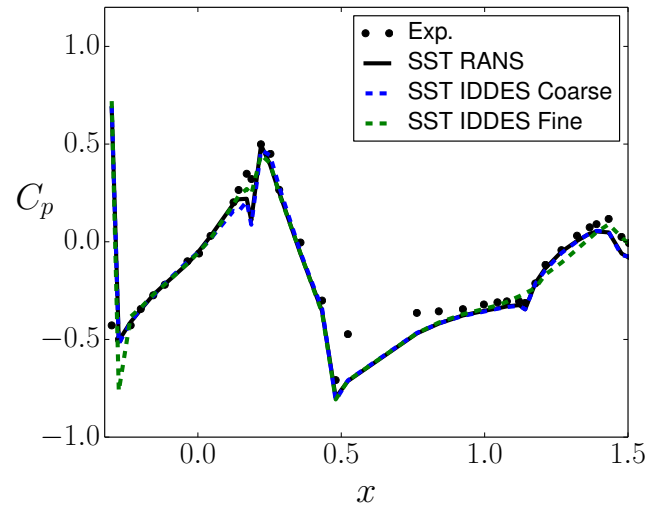

(a)

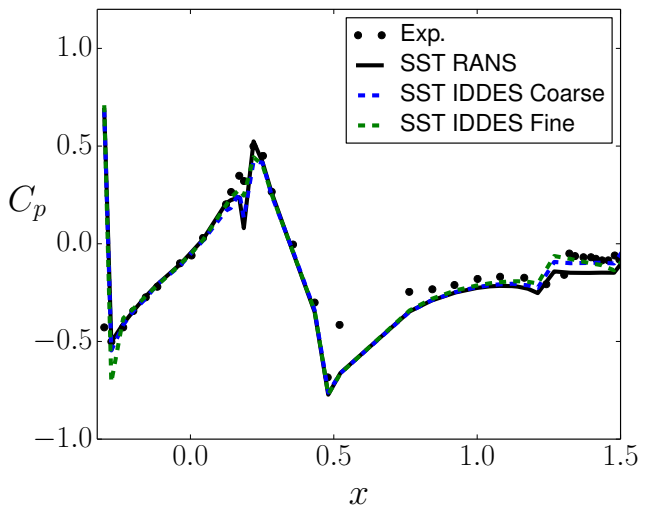

(b)

Figure 24: (a) Mean Pressure Coefficient over the top of the Fastback configuration for each mesh using the SST IDDES model. (b) Mean Pressure Coefficient over the top of the Estate configuration for each mesh using the SST IDDES model.

\section{Acknowledgements}

The authors gratefully acknowledge the support from CD-adapco and also BETA-CAE Systems for providing the ANSA pre-processing tool. The authors gratefully acknowledge computational support from EPSRC for UK's National HPC Facility, ARCHER and Barcelona Supercomputing Centre (BSC) for time on Mare Nostrum. This work also made use of the facilities of N8 HPC provided and funded by the N8 consortium and EPSRC (Grant No.EP/K000225/1). The Centre is co-ordinated by the Universities of Leeds and Manchester. Finally the authors would like to thank the Institute of Aerodynamics and Fluid Mechanics at Technische Universitat Munchen for providing the CAD data of the DrivAer models.

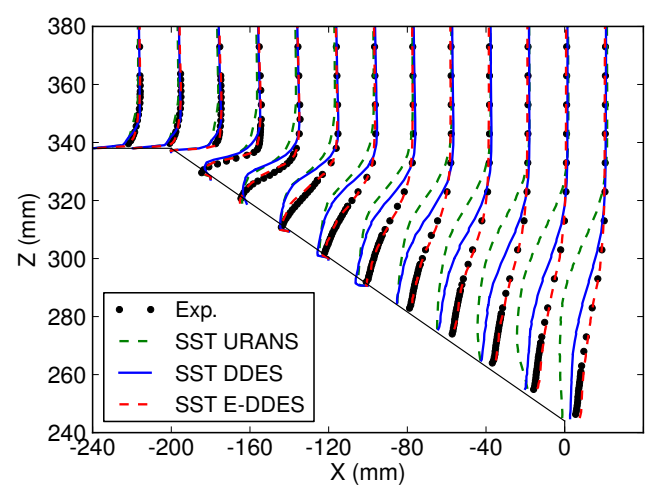

(a)

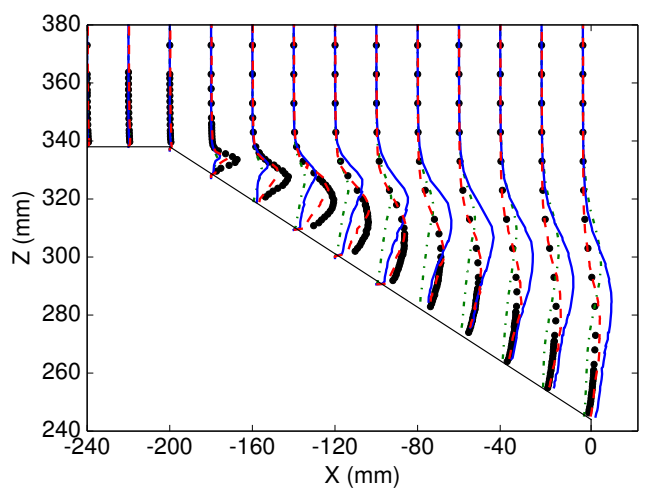

(b)

Figure 25: (a) Mean Streamwise velocity along the Ahmed car body for the SST-URANS, SST-DDES and SST-E-DDES models. (b) Mean Turbulent Kinetic Energy along the Ahmed car body for the SST-URANS, SST-DDES and SST E-DDES models. 


\section{References}

[1] Ahmed SR, Ramm G, Faltin G. Some salient features of the time averaged ground vehicle wake. SAE-Paper 840300 1984;.

[2] Lienhart H, Becker S. Flow and turbulent structure in the wake of a simplified car model. SAE 2003;01(1):0656.

[3] Heft AI, Adams NA. Experimental and numerical investigation of the drivaer model. In: Proceedings of the ASME 2012 Fluids Engineering Summer meeting. 2012, p. 1-11.

[4] Heft AI, Indinger T, Adams NA. Introduction of a New Realistic Generic Car Model for Aerodynamic Investigations. SAE 2012;doi:10.4271/2012-01-0168.

[5] Spalart PR, Allmaras SR. A one-equation turbulence model for aerodynamic flows. La Recherche Aerospatiale 1994;1:5-21.

[6] Spalart PR, Deck S, Shur ML, Squires KD, Strelets MK, Travin A. A New Version of Detached-eddy Simulation, Resistant to Ambiguous Grid Densities. Theor Comput Fluid Dynam 2006;20(3):181-95. doi:10.1007/s00162-006-0015-0.

[7] Shih T, Liou WW, Shabbir A, Yang Z, Zhu J. A new k-epsilon eddy viscosity model fo high Reynolds number turbulent flows. Computers \& Fluids 1995;24(3):227-38.

[8] Gritskevich MS, Garbaruk AV, Menter FR. Fine-tuning of DDES and IDDES formulations to the k-w Shear Stress Transport model. In: 4th European Conference for Aerospace Sciences EUCASS4. 2011, p. 1-10.

[9] Menter FR. Two-Equation Eddy-Viscosity Turbulence Models for Engineering Applications. AIAA 1994;32(8):1598-605.

[10] Billard F, Laurence D. A robust $\mathrm{k} \epsilon / \mathrm{k}$ elliptic blending turbulence model applied to near-wall, separated and buoyant flows. International Journal of Heat and Fluid Flow 2012;33(1):45-58. doi:10.1016/j.ijheatfluidflow.2011.11.003.

[11] Shur ML, Spalart PR, Strelets MK, Travin AK. A hybrid RANS-LES approach with delayed-DES and wall-modelled LES capabilities. International Journal of Heat and Fluid Flow 2008;29(6):1638-49. doi:10.1016/j.ijheatfluidflow.2008.07.001.

[12] Lardeau S, Manceau R. Computations of complex flow configurations using a modified Elliptic-Blending Reynolds-Stress Model. In: ETMM10: 10th International ERCOFTAC Symposium on Engineering. 2014,.

[13] Fröhlich J, von Terzi D. Hybrid LES/RANS methods for the simulation of turbulent flows. Progress in Aerospace Sciences $2008 ; 44: 349-77$.

[14] Mockett C. A comprehensive study of detached-eddy simulation. Ph.D. thesis; TUB; 2009.

[15] Wray A. Unpublished DNS data. Available on AGARD database Test Cases for the Validation of Large- Eddy Simulations of Turbulent Flows. 1997.

[16] Fournier Y, Bonelle J, Moulinec C, Shang Z, Sunderland A, Uribe J. Optimizing Code Saturne computations on Petascale systems. Computers and Fluids 2011;45(1):103-8. doi:10.1016/j.compfluid.2011.01.028.

[17] Ashton N, Prosser R, Revell A. A hybrid numerical scheme for a new formulation of delayed detached-eddy simulation (DDES) based on elliptic relaxation. In: Journal of Physics: Conference Series; vol. 318. 2011, p. 042043. doi:10.1088/1742-6596/318/4/042043.

[18] Schwamborn D, Strelets M. ATAAC An EU-Project Dedicated to Hybrid RANS-LES methods. In: Progress in Hybrid RANS-LES Modelling; vol. 117 of Notes on Num. Fluid Mech. and Multidisc. Design. 2012, p. 59-75.

[19] Ashton N, Revell A. Key factors in the use of DDES for the flow around a simplified car. International Journal of Heat and Fluid Flow 2015;54:236-49. URL: http://linkinghub.elsevier.com/retrieve/pii/S0142727X15000673. doi:10.1016/j.ijheatfluidflow.2015.06.002.

[20] Jakirlić S, Jester-Zurker R, Tropea C. Report on 9th ERCOFTAC/IAHR/COST Workshop on Refined Turbulence Modelling. In: ERCOFTAC Bulletin. Darmstadt University of Technology; 2002, p. 36-43.

[21] Manceau R, Bonnet JP, Leschziner M, Menter FR. 10th Joint ERCOFTAC(SIG-15)/IAHR/QNET-CFD Workshop on Refined Flow Modelling. Universite de Poitiers; 2002,

[22] Serre E, Minguez M, Pasquetti R, Guilmineau E, Deng GB, Kornhaas M, et al. On simulating the turbulent flow around the Ahmed body: A FrenchGerman collaborative evaluation of LES and DES. Computers $\backslash \&$ Fluids 2013;78:10-23. doi:10.1016/j.compfluid.2011.05.017.

[23] Mathey F, Cokljat D. Zonal multi-domain RANS/LES simulation of airflow over the Ahmed body. In: Rodi W, Mulas M, editors. Engineering turbulence modelling and experiments (ETMM 6). 2005, p. 647-56. 
[24] Caridi D, Cokljat D, Schuetze J, Lechner R. Embedded Large Eddy Simulation of Flow around the Ahmed Body. SAE World Congress 2012; doi:10.4271/2012-01-0587.

[25] Haase W, Aupoix B, Bunge U, Schwamborn D, editors. FLOMANIA - A European Initiative on Flow Physics Modelling; vol. 94 of Notes on Numerical Fluid Mechanics and Multidisciplinary Design. Springer-Verlag; 2006.

[26] Haase W, Braza M, Revell A. DESider - A European Effort on Hybrid RANS-LES Modelling; vol. 103 of Notes on Numerical Fluid Mechanics and Multidisciplinary Design. Springer-Verlag; 2007.

[27] Ashton N, Revell A, Poletto R. Grey-area mitigation for the Ahmed car body using embedded DDES. In: Progress in Hybrid RANS-LES Modelling. Notes on Num. Fluid Mech.; 2014,

[28] Hinterberger C, Garcia-Villalba M, Rodi W. Large eddy simulation of flow around the Ahmed body. In: The Aerodynamics of Heavy Vehicles: Trucks, Buses, and Trains; vol. 19 of Lecture notes in applied and computational mechanics. Springer; 2004,.

[29] Minguez M, Pasquetti R, Serre E. High-order large-eddy simulation of flow over the Ahmed body car model. Physics of Fluids 2008;20(9):095101. doi:10.1063/1.2952595.

[30] Krajnovic S, Davidson L. Large-eddy simulation of the flow around simplified car model. In: SAE World Congress. Detroit; 2004,.

[31] Kapadia S, Roy S, Vallero M, Wurtzler K, Forsythe J. Detached-Eddy Simulation over a reference ahmed car model. In: AIAA 41th Aerospace Sciences Meeting and Exhibit. Reno Nevada: AIAA; 2003,

[32] Guilmineau E, Deng G, Wackers J. Numerical simulation with a DES approach for automotive flows. Journal of Fluids and Structures 2011;27(5-6):807-16. URL: http://dx.doi.org/10.1016/j.jfluidstructs.2011.03.010. doi:10.1016/j.jfluidstructs.2011.03.010.

[33] Archambeau F, Mechitoua N, Sakiz M. A finite volume method for the computation of turbulent incompressible flows - industrial applications. Int J Finite Volumes 2004;1:1-62.

[34] Travin A, Shur M, Strelets M, Spalart PR. Physical and numerical upgrades in the detached-eddy simulation of complex turbulent flows. In: Proceedings of the 412th Euromech Colloquium on LES and Complex Transitional and Turbulent Flows. Munich; 2000,

[35] Peters BC, Uddin M, Bain J, Charlotte UNC, Engineering M. Simulating DrivAer with Structured Finite Difference Overset Grids. SAE Paper 2015-01-1536 2015; doi:10.4271/2015-01-1536.Copyright.

[36] Guilmineau E. Numerical Simulations of Flow around a Realistic Generic Car Model. SAE International Journal of Passenger Cars Mechanical Systems 2014;7(2):2014-01. URL: http://papers.sae.org/2014-01-0607/. doi:10.4271/2014-01-0607.

[37] Launder BE, Reece GJ, Rodi W. Progress in the development of a Reynolds stress turbulence closure. Journal of Fluid Mechanics 1975;68:537-66.

[38] Rumsey CL, Jeyapaul E. Pressure-strain and near-wall modeling for two-dimensional separated flows. In: Stanford Summer School. 2009; $2012,$.

[39] Poletto R, Craft T, Revell A. A New Divergence Free Synthetic Eddy Method for the Reproduction of Inlet Flow Conditions for LES. Flow, Turbulence and Combustion 2013;:519-39doi:10.1007/s10494-013-9488-2. 